\title{
Functional Genomics of the Rapidly Replicating Bacterium Vibrio Natriegens by CRISPRi
}

\section{Citation}

Lee, Henry H., Nili Ostrov, Brandon G. Wong, Michaela A. Gold, Ahmad S. Khalil, and George M. Church.2019. Functional Genomics of the Rapidly Replicating Bacterium Vibrio Natriegens by CRISPRi. Nature Microbiology 4, no. 7: 1105-113.

\section{Permanent link}

http://nrs.harvard.edu/urn-3:HUL.InstRepos:42083015

\section{Terms of Use}

This article was downloaded from Harvard University's DASH repository, and is made available under the terms and conditions applicable to Other Posted Material, as set forth at http:// nrs.harvard.edu/urn-3:HUL.InstRepos:dash.current.terms-of-use\#LAA

\section{Share Your Story}

The Harvard community has made this article openly available.

Please share how this access benefits you. Submit a story. 


\section{Functional genomics of the rapidly replicating bacterium 2 Vibrio natriegens by CRISPRi}

Henry H. Lee ${ }^{1^{*}, \dagger}$, Nili Ostrov ${ }^{1^{*}}$, Brandon G. Wong ${ }^{2}$, Michaela A. Gold ${ }^{1}$, Ahmad S. Khali1 ${ }^{2,3}$, George M. Church ${ }^{1,3, \dagger}$

\section{Author affiliations}

${ }^{1}$ Department of Genetics, Harvard Medical School, Boston, Massachusetts 02115, USA.

${ }^{2}$ Department of Biomedical Engineering and Biological Design Center, Boston University, Boston, Massachusetts 02215, USA.

${ }^{3}$ Wyss Institute for Biologically Inspired Engineering, Harvard University, Boston, Massachusetts 02115, USA.

*Equal contribution

${ }^{\dagger}$ Correspondence: gchurch@genetics.med.harvard.edu, hhlee@genetics.med.harvard.edu

\section{Main text}

The fast growing Gram-negative bacterium Vibrio natriegens is an attractive microbial system for molecular biology and biotechnology due to its remarkable short generation time ${ }^{1,2}$ and metabolic prowess ${ }^{3,4}$. However, efforts to uncover and utilize the mechanisms underlying its rapid growth are hampered by the scarcity of functional genomic data. Here, we develop a pooled genome-wide CRISPR interference (CRISPRi) screen to identify a minimal set of genes required for rapid wild-type growth. Targeting 4,565 (99.7\%) of predicted protein-coding genes, our screen uncovered core genes composed of putative essentials and growth-supporting genes which are enriched for respiratory pathways. We found $96 \%$ of core genes to be located on the larger chromosome 1, with growth-neutral duplicates of core gene located primarily on chromosome 2. Our screen also refines metabolic pathway annotations by distinguishing functional biosynthetic enzymes from those predicted based on comparative genomics. Taken together, this work provides a broadly applicable platform for high-throughput functional genomics to accelerate biological studies and engineering of $V$. natriegens.

We began by exploring laboratory culturing conditions which support rapid and consistent $V$. natriegens growth. We opted for Lysogeny Broth supplemented with $3 \%(\mathrm{w} / \mathrm{v})$ final sodium chloride (LB3), which approximates ocean salinity and provides consistent growth (Supplementary Figure 1, Supplementary Table 1). At $37^{\circ} \mathrm{C}$, we measured V. natriegens' generation time to be 15.2 minutes in bulk culture and 14.8 minutes using single-cell, time-lapse microscopy (Figure 1a-b, Supplementary Movie 1, Supplementary Figure 1, Supplementary Figure 2). Longer generation time (30.8 minutes) was observed in minimal glucose media supplemented with $2 \%(\mathrm{w} / \mathrm{v})$ sodium chloride (Supplementary Figure 1). These generation times are comparable with other studies ${ }^{3-5}$. 
We then produced two closed circular chromosomes of 3.24 Mb (chromosome 1; chr1) and 1.92 $\mathrm{Mb}$ (chromosome 2; chr2) by de novo sequence assembly, followed by annotation using $\mathrm{RAST}^{7}$ (RefSeq NZ_CP009977-8, Figure 1c, Supplementary Table 2, Supplementary Table 3, Methods) ${ }^{6}$. We identified 4,578 open reading frames (ORFs), of which $\sim 63 \%$ reside on chr1 and $\sim 37 \%$ on chr2. Consistent with the broad metabolic capacity described for Vibrio spp. ${ }^{8}$, nearly half of all annotated ORFs are involved in carbohydrates, RNA and protein metabolism (Figure 1d). $V$. natriegens genome carries 11 rRNA operons and 129 tRNA genes, more than $V$. cholerae N16961 (8 and 98, respectively) ${ }^{9}$ or E. coli MG1655 (7 and 87, respectively) ${ }^{10}$. We further identified 36,599 putative methylated adenine residues at GATC motifs. Finally, we mapped the origin and terminus of each chromosome by quantifying sequencing coverage of actively dividing cells ${ }^{11}$, and found a single bidirectional origin on each chromosome (Figure 1e) which displayed high sequence similarity to other Vibrio spp. (Supplementary Figure 3) ${ }^{9}$.

We next sought to develop genetic tools to enable high-throughput assessment of gene functionality. While transposon mutagenesis is commonly used to generate single gene mutant libraries $^{12}$, low insertion efficiency prohibited scalable saturation mutagenesis in $V$. natriegens (Supplementary Table 8, Supplementary Discussion). Instead, we turned to CRISPR/Cas9 which has been demonstrated in well-studied model organisms ${ }^{13-16}$ but has not been scaled for functional genomics in emerging microbial systems.

To test CRISPR/Cas9 functionality, we created a reporter $V$. natriegens strain expressing GFP, and tested the Streptococcus pyogenes Cas9 nuclease with or without GFP-targeting guide RNA (gRNA) (plasmid pRSF-paraB-spCas9-gRNA, Supplementary Figures 5-7, Supplementary Table 4). No transformants were detected following electroporation of Cas9 nuclease with a GFP-targeting gRNA, even without induction of Cas9 expression (Supplementary Figure 9). This result indicates significant toxicity due to double-stranded breaks and undetectable levels of non-homologous end joining (NHEJ). We thus concluded that the CRISPR/Cas9 system alone cannot be used for efficient generation of mutants by NHEJ.

Transcriptional inhibition of gene expression by CRISPR inhibition (CRISPRi) relies on the nuclease-deficient variant dCas9 to block RNA polymerase activity ${ }^{15}$. Based on design rules elucidated in other model bacteria ${ }^{14,15}$, we designed guides targeting the template and nontemplate strand proximal to GFP transcriptional start site and measured fluorescence in the presence of dCas9 (plasmid pRSF-paraB-dCas9-gRNA, Supplementary Table 4). Consistent with previous studies, we observed greater inhibition when targeting the non-template strand ( $>13$-fold, Figure 2a). As with Cas9 nuclease, induction of dCas9 was not required for activity and led to longer lag phase indicative of marginal toxicity (Supplementary Figure 9). We thus opted to use the presence and absence of the dCas9 plasmid in lieu of induction for subsequent experiments. 
For high-throughput screening of single-gene inhibition by CRISPRi, we transformed a pool of gRNA plasmids such that each cell carries dCas9 and a single gRNA targeting inhibition of one protein coding gene. The library was grown as a competitive culture and the abundance of each gRNA was determined by sequencing of gRNA cassettes. Since the abundance of gRNA reflects the fitness cost of reduced gene expression, we expect rapid depletion of gRNAs which target genes that, when inhibited, impair cellular growth.

We initially used a small library of five gRNAs to test the feasibility of a pooled CRISPRi approach. gRNAs were selected for inhibition of one putative essential gene ( $l p t F_{V n}$, a homolog of an essential $E$. coli gene), one putative non-essential $V$. natriegens gene $\left(f \lg C_{V n}\right)$, and two control genes absent from the genome (GFP and E.coli gene lptF). All gRNAs plasmids (vector pCTX-R6K-gRNA, Supplementary Figure 8) were co-transformed into V. natriegens bearing dCas9 (plasmid pdCas9-bacteria ${ }^{15}$ ). Plasmids were extracted and sequenced to determine the abundance of each gRNA over three hours of competitive growth. We observed a significant reduction in abundance of the gRNA targeting the putative essential $l p t F_{V n}$ gene, while all other guides remained relatively unchanged (Figure 2b, Supplementary Figure 10). These results demonstrate that gRNA abundance can be used as proxy for a gene's fitness in pooled competitive growth.

Next, we scaled up our gRNA library to cover all predicted ORFs in the $V$. natriegens genome. Transcriptional knockdown of a gene by CRISPRi can be achieved by targeting either its transcriptional start site, which blocks initiation, or its coding region, which blocks elongation ${ }^{15}$. As transcriptional start sites have not yet been mapped in this organism, we designed gRNAs targeting the non-template strand proximal to each gene's coding region. We created a library of 13,567 unique gRNAs to target 4,565 (99.7\%) RAST-predicted protein-coding genes. Our library includes up to three unique gRNAs per gene, with $88 \%$ of all guides within the first $20 \%$ of the coding sequence (Figure 2c, Supplementary Table 5). Library screens were performed in LB3, M9-Glucose, or M9-Sucrose for 8 hours (Figure 2d, Supplementary Figure 11). To estimate fitness for each gene from its targeting gRNAs, we generated a beta score and statistical significance using MAGeCK's maximum likelihood estimation (MLE) ${ }^{17}$ (Supplementary Figure 5). A negative beta score indicates depletion of a gRNA under the tested condition.

Using sucrose as the sole carbon source, we identified fourteen unique genes with a significant negative beta scores $(F D R \leq 0.05)$ (Figure 3a, Supplementary Table 5). These include phosphoglucomutase, several membrane transport proteins, and sucrose-6-phosphate hydrolase, a key component of intracellular sucrose catabolism. As only one of two annotated sucrose-6phosphate hydrolase genes was identified in our screen, we generated in-frame deletion of both genes and measured each under non-competitive growth conditions. Consistent with CRISPRi results, we found the sucrose-6-phosphate hydrolase located on chr1 (PEG.1381, $\beta_{\text {suc }}=-1.6907$ ), 
121 but not the one located on chr2 (PEG.3071, $\beta_{\mathrm{suc}}=0.12$ ), was required for growth in M9-sucrose media (Figure 3b). This demonstrates the utility of our approach for identifying functionally relevant genes.

Overall, our glucose and sucrose minimal media screen yielded 143 unique genes (FDR $\leq 0.05$ ) enriched in RAST subcategories for amino acid and vitamin biosynthesis (Figure 3c, Supplementary Figure 12a). When mapped onto the respective pathways, this set can be used to distinguish functional genes from a larger set of computationally assigned biosynthetic enzymes $^{7,18}$. For example, of all predicted L-leucine biosynthetic genes, only one of two acetolactate synthase complexes (PEGs 2236, 2237 and 2615, 2616) and one of two isopropylmalate synthases (PEGs 2243 and 3818) was identified as functional in our screen (Figure 3d). Similar results were obtained for tryptophan and isoleucine biosynthetic pathways. Moreover, we show functional enzymes are not always the most differentially expressed (Figure 3d, Supplementary Figure 12b,c). Thus, while transcriptional enrichment corroborates the importance of amino acid biosynthesis pathways in minimal media (Supplementary Table 7), expression level is not sufficient to infer gene functionality.

We next sought to identify a minimal set of genes necessary for rapid wild type growth in rich media (LB3). In our first experiment, we found 646 genes with significant negative beta score (Figure 3a). When repeated, the same screen resulted in a slightly larger number of significant genes (735), though further passaging of the culture yielded only 648 significant negative scoring genes. Of these, 587 (92.4\%) overlap between the two experiment (Figure 4a, Supplementary Figure 13). Given the high overlap, these data indicate that passaging reduces variability and provides more stringent results. We thus define 587 genes identified in both experiments as 'core genes' which are required for rapid growth in rich media (Supplementary Table 6).

We further analyzed the passaging experiments for evidence of CRISPRi escape by examining 100 genes which were identified as significant in the first, but not the last passage (Supplementary Figure 13e, Supplementary Table 5). We expect CRISPRi escape to result in increased beta score over passages. However, the majority of these genes $(73 \%)$ displayed a decrease in beta score, suggesting that rather than escaping dCas9 inhibition, they likely lost significance due to increase in the total number of low scoring genes (Supplementary Figure 13f). Of the remaining 27 genes which increase in beta score over passaging, only seven were altered by more than $20 \%$ (Supplementary Figure 14a). For these, we found one of three gRNA was repeatedly elevated in the third passage (Supplementary Figure 14b), indicating that increase in beta score is due to an inefficient or promiscuous gRNA sequence rather than escape. majority are located on chr1 (96.0\%, 564 genes) (Supplementary Table 6). Only 16 core genes $(2.7 \%)$ were annotated as hypothetical proteins. We found the median expression for core genes 
was 5.6-fold higher than non-core genes (160.71 and 28.56 median transcripts per million TPM, respectively). To assess essentiality, we compared the core set to genes previously identified by high-density transposon mutagenesis in the well-studied Vibrio cholerae genome ${ }^{19}$. We found 278 homologous genes, corresponding to $84.4 \%$ of the 329 mappable essential genes in $V$. cholerae, which we designate putative essential genes in $V$. natriegens (Figure $4 \mathbf{b}$, Supplementary Table 6).

Similar to other Vibrio spp. ${ }^{20}$, the vast majority of putative essentials are located on chr1 (272 of 278 genes). Putative essentials were statistically enriched for RAST categories including 'Protein metabolism' and 'Cell division and cell cycle', which include ribosomal proteins, tRNA synthetases, and DNA polymerase (Figure 4c, Supplementary Figure 15). For example, 49 of 55 total ribosomal proteins are included in the core set, of which $44(89.8 \%)$ are in agreement with essentiality in $V$. cholerae and $37(75.5 \%)$ are in agreement with essentiality in $E$.coli, as determined by transposon mutagenesis ${ }^{19}$ and gene deletions ${ }^{21}$, respectively (Figure 4d, Supplementary Table 6). Other notable essentials include DNA adenine methylase dam $^{22}$ and nadE, a NAD + synthase gene implicated in the conversion of chr2 from a megaplasmid into a critical component of the Vibrio genome ${ }^{23}$. Putative essential genes, which were all actively transcribed, showed lower beta scores and higher transcription levels than other core genes (Figure 4e-f, Supplementary Figure 15a), in line with reports of essential gene expression in other organisms ${ }^{24}$.

Non-essential core genes (309 of 587 genes) were uniquely enriched for the RAST category 'Respiration' (Figure 4c, Supplementary Figure 15b). This set of 'growth-supporting' genes include the Rnf electron transport complex, cytochrome $\mathrm{C}$ oxidase biogenesis enzymes, and the $\mathrm{Na}+-\mathrm{NQR}$ respiration complex implicated in aerobic respiration in halophilic bacteria ${ }^{25}$ (Supplementary Table 6). These pathways are likely responsible for the high oxygen uptake and highly active electron transport chain activity ${ }^{3}$ required to maintain rapid cell division. Others, such as quorum sensing regulator $h f q^{26}$, may determine the rapid growth rate observed in highly dilute cultures ${ }^{4}$. Further investigation of growth-supporting genes will refine our understanding of the mechanisms underlying rapid $V$. natriegens growth.

Interestingly, we found that $14.3 \%$ of core genes (84 of 587 genes) - including Tyrosyl-tRNA synthetase, heat shock proteins GroES/EL, DNA polymerase I and chaperone DnaK - share identical annotation with one or more genes. Duplicates are distributed similarly between essential and growth-supporting core genes (39 and 45, respectively) and most have nonsignificant negative beta scores, suggesting they are unlikely to be functionally redundant (Supplementary Figure 16a). Only five genes were found where both duplicates are independently identified in our screen, including EF-Tu, Geranylgeranyl pyrophosphate synthetase, MotA/TolQ/ExbB proton channel family protein, Ferric iron ABC transporter ATPbinding protein, and a benzoquinol hydroxylase (Supplementary Table 6). We found $60.3 \%$ of 
core genes with duplicate (53 of 84 genes) are located on chr1 with the duplicate located on chr2 (Figure 4g), contributing to the relatively large size of $\operatorname{chr} 2$ in $V$. natriegens compared to other Vibrio spp. ${ }^{20}$. Duplicated genes, previously reported in $V$. cholerae $^{19}$, may represent heterofunctional homologs or pseudogenes. Transcriptional profiling shows the median TPM for core genes is $\sim 17$-fold higher than that of duplicates (125.6 and 7.4, respectively), suggesting that expression of duplicates may be silenced under tested conditions (Supplementary Figure 16b). Further insight into the genesis and role of these gene duplications may benefit from studies of interchromosomal interactions ${ }^{27}$ in Vibrio genomes.

Interpretation of pooled CRISPRi results should consider potential caveats. First, metabolite cross feeding, such as amino acid exchange, may obscure the effect of inhibition for critical pathway enzymes ${ }^{28}$. Second, as CRISPRi relies on reducing RNA polymerase elongation, inhibition of upstream genes in an operon may result in erroneous determination of essentiality for downstream genes ${ }^{14}$. Identification of transcriptional start sites and operon structures, currently unavailable for $V$. natriegens, will help to better address polar effects. Third, changes to the statistical significance threshold may alter the list of functionally relevant genes. Lastly, screen results may be affected by dCas9-specific toxicity, as observed for recQ helicase (Supplementary Discussion, Supplementary Figure 17). Additional studies using in-frame deletions are warranted to stringently evaluate the phenotype of single gene knockout.

Nevertheless, screening sensitivity and specificity may be further increased by improvements to the gRNA library and dCas9 protein. While three gRNAs were used to mitigate inefficient gene knockdown, we cannot exclude the possibility that inhibition levels vary between genes since the knockdown efficiency for each gRNA was not measured. Future studies may utilize individually optimized gRNAs to ensure equal inhibition between genes or increase the number of gRNAs per gene for robust statistical inference. The library can also be expanded to target non-coding elements, such as RNA or intergenic regions, to reveal functional genomic features not captured by computational annotation. Additional design principles for CRISPRi libraries ${ }^{16,29}$ may be used to improve gRNA binding specificity or reduce potential sequence-specific toxicity ${ }^{29}$. Furthermore, gene dosage effects can be explored by tuning transcriptional knockdown via engineering of $V$. natriegens promoters or synthetic dCas 9 with reduced toxicity ${ }^{30}$. Overall, this pooled approach can be utilized to screen for genotypes underlying any growth-linked phenotype to accelerate our understanding of this organism. More broadly, we envision the use of pooled genome-wide CRISPRi screens will enable rapid and high-throughput functional annotation of diverse bacteria.

Taken together, we provide initial genome-wide experimental annotations for $V$. natriegens which can inform targeted studies of its fast growth and metabolic capacity. These findings may also provide a starting point for large-scale genome engineering. For example, core genes can be used as a basis for probing the limits of codon reassignment. Furthermore, beta scores can 
241 provide valuable experimental guidance for construction of a fast growing minimal genome by 242 bottom up construction or large-scale deletion ${ }^{2}$. With only $2.1 \%$ of putative essential genes 243 located on chr2 (Figure 4e, Supplementary Table 6), spatial distribution of core genes presents 244 intriguing opportunities for rational genome design, such as consolidating of functional genes to 245 chromosome 1 and re-purposing of chromosome 2 as an artificial chromosome. Further 246 investigation of this unique organism will facilitate its advancement as a versatile bacterial 247 system for research and biotechnology.

248 


\section{Supplementary Materials}

250 Supplementary Tables $1-8$

251 Supplementary Figures $1-17$

252 Supplementary Movie 1

254 Correspondence to gchurch@genetics.med.harvard.edu, hhlee@genetics.med.harvard.edu

\section{Acknowledgements}

257 We would like to acknowledge Javier Fernández Juárez, Jun Teramoto, Michael Mee, Andrew

258 Camilli, and John Aach for helpful comments and discussions. Ahmad Khalil would additionally 259 like to acknowledge Christopher Mancuso and Madeleine Joung. We thank Lyubov Golubeva 260 for the pRSF plasmid, Brigid Davis and Matthew Waldor for Vibrio cholerae strains O395, 261 BAH-2, and pCTX-Km and pCTX-Ap plasmids, Victor de Lorenzo for pBAM1 plasmid, D. 262 Ewen Cameron and John Mekalanos for pTnFGL3 plasmid and Barry Wanner for BW29427 263 strain. This work was supported by Department of Energy Grant DE-FG02-02ER63445 (to 264 G.M.C.), AWS Cloud Credits for Research program (to H.H.L.), and a National Science 265 Foundation CAREER Award MCB-1350949 (to A.S.K.).

267 Author contributions

268 H.H.L. and N.O. designed, performed experiments, analyzed data, and wrote the paper. B.W. and

269 A.S.K. designed and performed single-cell microfluidics experiments, and provided input on the 270 paper. M.A.G. contributed to the electroporation experiments and formulated recovery media. 271 G.M.C. supervised the project.

274 H.H.L., N.O., and G.M.C. have filed patents related to this work. 
Strains used in this study

Wild type $V$. natriegens (ATCC 14048) was used in this study. GFP reporter strain was constructed by genomic integration of a pLtetO-gfp-kanamycin cassette using the mariner transposon system described under methods.

\section{Growth Media}

Unless denoted, LB3, Lysogeny Broth with 3\% (w/v) final $\mathrm{NaCl}$, is used as standard rich media. We prepare this media by adding 20 grams of $\mathrm{NaCl}$ to 25 grams of LB Broth - Miller (Fisher BP9723-500). Media are formulated according to manufacturer instructions and supplemented with $1.5 \%(\mathrm{w} / \mathrm{v})$ final Ocean Salts (Aquarium System, Inc.) to make high salt versions of Brain Heart Infusion (BHIO), Nutrient Broth (NBO), and Lysogeny Broth (LBO). No additional salts were added to Marine Broth (MB). Minimal M9 media was prepared according to manufacturer instruction. For culturing $V$. natriegens, $2 \%(\mathrm{w} / \mathrm{v}) \mathrm{NaCl}$ was added to $\mathrm{M} 9$ (in addition to the preincluded $0.5 \mathrm{~g} / \mathrm{L} \mathrm{NaCl}$ ) based on the screen of $\mathrm{NaCl}$ concentrations in rich media. Carbon sources were added as indicated to $0.4 \%(\mathrm{v} / \mathrm{v})$ final. SOC3 media is composed of 5 grams of yeast extract, 20 grams tryptone, 30 grams sodium chloride, 2.4 grams magnesium sulfate, and $0.4 \%(\mathrm{w} / \mathrm{v})$ final glucose. Antibiotic concentrations used for plasmid selection in $V$. natriegens: ampicillin/carbenicillin $100 \mu \mathrm{g} / \mathrm{ml}$, kanamycin $75 \mu \mathrm{g} / \mathrm{ml}$, chloramphenicol $5 \mu \mathrm{g} / \mathrm{ml}$, spectinomycin $100 \mu \mathrm{g} / \mathrm{ml}$. E. coli experiments were performed in standard LB media and M9.

Culturing and glycerol stock conditions

An inoculation of $-80^{\circ} \mathrm{C}$ frozen stock of $V$. natriegens can reach stationary phase after 5 hours when incubated at $37^{\circ} \mathrm{C}$. Prolonged overnight culturing ( $>15$ hours) at $37^{\circ} \mathrm{C}$ may lead to an extended lag phase upon subculturing. Routine overnight culturing of $V$. natriegens was performed for $8-15$ hours at $37^{\circ} \mathrm{C}$ or $12-24$ hours at room temperature. Unless otherwise indicated, E. coli cells used in this study were K-12 subtype MG1655 cultured overnight ( $>10$ hours) at $37^{\circ} \mathrm{C}$. V. cholerae $\mathrm{O} 395$ was cultured overnight $\left(>10\right.$ hours) in $\mathrm{LB}$ at $30^{\circ} \mathrm{C}$ or $37^{\circ} \mathrm{C}$ in a rotator drum at $150 \mathrm{rpm}$. To prepare $V$. natriegens cells for $-80^{\circ} \mathrm{C}$ storage, an overnight culture of $V$. natriegens is washed in fresh media before storing in glycerol. Cultures were centrifuged for 1 minute at 20,000 rcf and the supernatant was removed. The cell pellet was resuspended in fresh LB3 media and glycerol was added to $20 \%$ final concentration. The stock is quickly vortexed and stored at $-80^{\circ} \mathrm{C}$. Bacterial glycerol stocks stored in this manner are viable for at least 5 years.

\section{Bulk measurements of generation time} well plates with continuous orbital shaking and optical density (OD) measurement at 600nm taken every 2 minutes. Overnight cells were washed once in fresh growth media, then subcultured by at least 1:100 dilution. To assay $V$. natriegens growth in different rich media, 
cells were cultured overnight from frozen stock into the respective media. To assay $V$. natriegens and E. coli growth in minimal media, cells were cultured overnight in LB3 and LB respectively, and subcultured in the appropriate test media. Generation times were calculated by linear regression of the log-transformed OD across at least 3 data points when growth was in exponential phase. To avoid specious determination of growth rates due to measurement noise, the minimal OD considered for analysis was maximized and the ODs were smoothed with a moving average window of 3 data points for conditions that were challenging for growth. Maximal growth rates were computed from generation times. Apparent lag times were estimated with a fitted model-free smoothed spline using "grofit" R package.

\section{Construction of microfluidics device or single cell imaging}

Microfluidic devices were used as tools to measure and compare growth rates of $E$. coli and $V$. natriegens. In these devices, cells are grown in monolayer and segmented/tracked in high temporal resolution using time-lapse microscopy. The cells are constricted for imaging using previously described Tesla microchemostat device designs, in which cell traps have heights that match the diameters of the cells, minimizing movement and restricting growth in a monolayer ${ }^{31}$. Different trapping heights of $0.8 \mu \mathrm{m}$ and $1.1 \mu \mathrm{m}$ were used for $E$. coli and $V$. natriegens, respectively. Microfluidic devices were fabricated with polydimethylsiloxane (PDMS/Sylgard 184, Dow Corning) using standard soft lithographic methods. Briefly, microfluidic devices were fabricated by reverse molding from a silicon wafer patterned with two layers of photoresist (one for the cell trap, another for flow channels). First, the cell trap layer was fabricated by spin coating SU-8 2 (MicroChem Corp.) negative resist at 7000 RPM and 6800 RPM for E. coli and $V$. natriegens, respectively, and patterned using a high resolution photomask (CAD/Art Services, Inc.). Next, AZ4620 positive photoresist (Capitol Scientific, Inc.) was spun onto the silicon wafer and aligned with another photomask for fabrication of $\sim 8 \mu \mathrm{m}$ tall flow channels (same for both organisms). Reverse-molded PDMS devices were punched and bonded to No. 1.5 glass coverslips (Fisher Scientific).

\section{Time-lapse microscopy and image analysis}

Cells were diluted down to $0.1 \mathrm{OD}_{600}$ from an overnight culture at optimal growth conditions and allowed to grow for an hour in the corresponding media conditions (e.g. temperature, salt concentration) before loading onto the device. Next, cells were loaded and grown on the device in the corresponding environmental conditions until the cell trap chambers filled. Temperature was maintained with a Controlled Environment Microscope Incubator (Nikon Instruments, Inc.). Media flow on device was maintained by a constant pressure of 5 psi over the course of the experiment after cell loading. During the experiment, phase contrast images were acquired every minute with a 100x objective (Plan Apo Lambda 100X, NA 1.45) using an Eclipse Ti-E inverted microscope (Nikon Instruments, Inc.), equipped with the "Perfect Focus" system, a motorized stage, and a Clara-E charge-coupled device (CCD) camera (Andor Technology). After the experiment, individual cells were segmented from the image time course using custom 
MATLAB (Mathworks, Natick, MA) software. Doubling time of cells was scored well before the density of the chamber impacted tracking and growth of cells. Results from repeat experiments on different days and devices were consistent (Data not shown).

Genome sequencing by Pacific Bioscience sequencing, de novo assembly, and annotation V. natriegens (ATCC 14048) was cultured for 24 hours at $30^{\circ} \mathrm{C}$ in Nutrient Broth with $1.5 \%$ $\mathrm{NaCl}$ according to ATCC instructions. Genomic DNA was purified (Qiagen Puregene Yeast/Bact. Kit B) and sequenced on a Single Molecule Real Time (SMRT) Pacific Biosciences RS II system (University of Massachusetts Medical School Deep Sequencing Core) using 120 minute movies on 3 SMRTCells. SMRTanalysis v2.1 on Amazon Web Services was used to process and assemble the sequencing data. The mean read length, after default quality filtering, was 4,407bp. HGAP3 with default parameters was used to assemble the reads which yielded 2 contigs. The contigs were visualized with Gepard and manually closed. The two closed chromosomes annotated using RAST under ID 691.12. The annotated genome is deposited in NCBI under Biosample SAMN03178087, GenBank CP009977-8, RefSeq NZ_CP009977-8. Base modification detection was performed on SMRTanalysis v2.1 with default setting and the closed genome as reference. Codon usage was calculated using EMBOSS cusp.

Mapping chromosomal origins and termini by Oxford Nanopore sequencing $V$. natriegens was cultured in LB3 and E. coli was cultured in LB. Both cultures were grown overnight at $37^{\circ} \mathrm{C}$. For stationary phase samples, $1 \mathrm{~mL}$ of each culture was collected for genomic DNA extraction. For exponential phase samples, each culture was subcultured and grown to $\mathrm{OD}_{600} \sim 0.4$ and $10 \mathrm{~mL}$ of each was collected for genomic DNA extraction. Genomic DNA was purified (Qiagen Puregene Yeast/Bact. Kit B). To maximize read length, $\sim 1 \mu \mathrm{g}$ of genomic DNA for each sample was used as input. 1D sequencing libraries were prepared, barcoded (SQKRAD002 and SQK-RBK001), and sequenced on the MinION with SpotON R9.4 flow cells for 48 hours. Cloud base-calling and sample demultiplexing was performed on Metrichor 1.4 .5 and FASTQ files prepared from FAST5 HDF files with a custom python script. Sequences were aligned to the reference genome using GraphMap 0.5.1. Coverage was computed with bedtools 2.26.0 and GC-skew computed using the iRep package.

Transcriptome profiling

Triplicate $V$. natriegens cultures were grown overnight from $-80^{\circ} \mathrm{C}$ stocks for each condition to be assayed: $30^{\circ} \mathrm{C}$ in $\mathrm{LB} 3,37^{\circ} \mathrm{C}$ in $\mathrm{LB} 3$ and $37^{\circ} \mathrm{C}$ in $\mathrm{M} 9$ high-salt media supplemented with $2 \%$ $(\mathrm{w} / \mathrm{v})$ final sodium chloride and $0.4 \%(\mathrm{w} / \mathrm{v})$ glucose. Each culture was subcultured in the desired conditions and grown to exponential phase $\left(\mathrm{OD}_{600}\right.$ 0.3-0.6). To collect RNA, $10 \mathrm{~mL}$ of each culture was stabilized with Qiagen RNAprotect Bacteria Reagent and frozen at $-80^{\circ} \mathrm{C}$. RNA extraction was performed with Qiagen RNeasy Mini Kit and rRNA depleted with Illumina RiboZero rRNA Removal Kit (Bacteria). Samples were spot-checked for RNA sample quality on an Agilent 2100 RNA 6000 Nano Kit to ensure that the RNA Integrity Number (RIN) was above 9. 
Sequencing libraries were prepared with the NEXTflex Rapid Directional qRNA-Seq Kit. Each sample was barcoded and amplified with cycle-limited real-time PCR with KAPA SYBR FAST. Resulting libraries were sequenced with MiSeq v3 150 to obtain paired end reads. Sequences were trimmed with cutadapt. Transcripts were quantified with Salmon 0.8.1 and counts were summarized with tximport for differential expression analysis with DESeq2.

\section{Construction and analysis of transposon mutant libraries}

To facilitate transposon mutagenesis, we engineered a suicide mariner-based transposon vector modified for insertion mapping by high-throughput sequencing ${ }^{12,32}$. Our conjugative suicide mariner transposon plasmid was propagated in BW29427, an E. coli with diaminopimelic acid (DAP) auxotrophy. BW29427 growth requires $300 \mu \mathrm{M}$ of DAP even when cultured in LB. Importantly, BW29427 does not grow in the absence of DAP, which simplifies counterselection of this host strain following biparental mating with $V$. natriegens. For conjugation from E. coli to $V$. natriegens, $24 \mathrm{~mL}$ of each strain was grown to OD 0.4, spun down, resuspended and plated on LB2 plates (Lysogeny Broth with $2 \%\left(\mathrm{w} / \mathrm{v}\right.$ ) final of sodium chloride) and incubated at $37^{\circ} \mathrm{C}$ for 60 minutes (see Supplementary Figure 4 for optimization of conjugation conditions). This conjugation time was chosen to minimize clonal amplification, based on optimization experiments using $100 \mathrm{uL}$ of each strain. The cells are recovered from the plate in $1 \mathrm{~mL}$ of LB3 media. The resulting cell resuspension is washed once in fresh LB3, resuspended to a final volume of $1 \mathrm{~mL}$, and plated on $245 \mathrm{~mm}$ x $245 \mathrm{~mm}$ kanamycin selective plates (Corning). Plates were incubated at $30^{\circ} \mathrm{C}$ for 12 hours to allow the formation of $V$. natriegens colonies. Colonies were scraped from each plate with $3 \mathrm{~mL}$ of LB3, gently vortexed, and stored as glycerol stock as previously described. No colonies were detected in control experiments with only BW29427 donor cells. A similar protocol was used to generate an E. coli transposon mutant library, except LB was used as the media at all steps.

For analysis of transposon mutant library, genomic DNA was extracted (Qiagen DNeasy Blood \& Tissue Kit), and digested with MmeI. To enrich for the fragment corresponding to the kanamycin transposon fragment, the digested genomic DNA was electrophoresed on a $1 \% \mathrm{TAE}$ gel and an area of the gel corresponding to approximately $1.2 \mathrm{~kb}$ was extracted. The resulting DNA fragment was sticky-end ligated to an adapter. PCR was used to selectively amplify the region around the transposon mosaic end and to add the required Illumina adapters. These amplicons were sequenced 1x50bp on a MiSeq. Since properly prepared amplicons contain 16 or $17 \mathrm{bp}$ of genomic DNA and 32 or 33bp of the ligated adapter, only those sequencing reads with the presence of the adapter were further analyzed. All adapters were trimming and the resulting genomic DNA sequences were aligned to the reference genome with Bowtie. Statistical enrichment of RAST categories were computed with the hypergeometric test and resulting pvalues were adjusted with Benjamini-Hochberg correction. Transposon mutagenesis data is 
For the E. coli Himar1 mutant library, we isolated $1.1 \times 10^{6}$ transconjugants, prepared Tn-Seq fragments as previously described, and analyzed by MiSeq ${ }^{12}$. We obtained $6.9 \times 10^{6}$ total reads, of which $1.6 \%$ mapped to the transposon plasmid; $98.3 \%$ of filtered reads were mapped to the genome. These insertions represent 107,723 unique positions, where $>10$ unique insertions were present in 3,169 out of 4,917 features. For the $V$. natriegens Himar1 mutant library we isolated $8.6 \times 10^{5}$ mutants. We obtained $6.1 \times 10^{6}$ reads, of which $36.4 \%$ mapped to the transposon plasmid; $97.2 \%$ of filtered reads were mapped to the genome. These insertions represent 4,530 unique positions, proportionally distributed between the two chromosomes where $>1$ unique insertions were found in 2,357 out of 4,940 features.

\section{Electroporation protocol for DNA transformation of $V$. natriegens} into growth media. Cells were harvested at $\mathrm{OD}_{600} \sim 0.4$ (1 hour growth when incubated at $37^{\circ} \mathrm{C}$ at $225 \mathrm{rpm}$ ) and pelleted by centrifugation at $3500 \mathrm{rpm}$ for $5 \mathrm{~min}$ at $4^{\circ} \mathrm{C}$. The pellet is washed three times using $1 \mathrm{ml}$ of cold $1 \mathrm{M}$ sorbitol and centrifuged at 20,000 ref for 1 minute at $4^{\circ} \mathrm{C}$. The final cell pellet was resuspended in $1 \mathrm{M}$ sorbitol at a 200 -fold concentrate of the initial culture. For long term storage, the concentrated competent cells were aliquoted in $50 \mu \mathrm{L}$ shots in pre-chilled tubes, snap frozen in dry ice and ethanol, and stored in $-80^{\circ} \mathrm{C}$ for future use. To transform, $50 \mathrm{ng}$ of plasmid DNA was added to $50 \mu \mathrm{L}$ of concentrated cells in $0.1 \mathrm{~mm}$ cuvettes and electroporated using Bio-Rad Gene Pulser electroporator at $0.4 \mathrm{kV}, 1 \mathrm{k} \Omega, 25 \mu \mathrm{F}$ and recovered in $1 \mathrm{~mL} \mathrm{LB} 3$ or SOC3 media for 45 minutes at $37^{\circ} \mathrm{C}$ at $225 \mathrm{rpm}$, and plated on selective media. Plates were incubated at least 6 hours at $37^{\circ} \mathrm{C}$ or at least 12 hours at room temperature.

\section{$\underline{\text { Plasmid construction }}$}

Routine cloning was performed by PCR of desired DNA fragments, assembly with NEB Gibson Assembly or NEBuilder HiFi DNA Assembly, and propagation in E. coli unless otherwise indicated. See Supplementary Table 4 for plasmids used in this work. Plasmid maps are provided in Supplementary Figure 5. We used pRSF for the majority of our work since it carries all of its own replication machinery and should be minimally dependent on host factors. For transformation optimizations, we constructed pRSF-pLtetO-gfp which constitutively expresses GFP due to the absence of the tetR repressor in both E. coli and V. natriegens. We engineered pCTX-R6K shuttle plasmid by fusing the pCTX-Km replicon, comprised of genes RstR and RstA, with the pir-dependent conditional replicon, R6k. After electroporation of this shuttle plasmid into $V$. natriegens, we were able to extract plasmid from a standard miniprep, demonstrating plasmid replication. To construct the conjugative suicide mariner transposon, we replaced the Tn5 transposase and Tn5 mosaic ends in pBAM1 with the mariner C9 transposase performed site-directed mutagenesis on both transposon mosaic ends to introduce an MmeI cutsite, producing the plasmid pMarC9 which is also based on the pir-dependent conditional 
replicon, R6k. We also constructed a transposon plasmid capable of integrating a constitutively expressing GFP cassette in the genome by inserting pLtetO-GFP with either kanamycin or spectinomycin in the transposon DNA. All plasmids carrying the R6k origin was found to replicate only in either BW29427 or EC100D $\mathrm{pir}^{+} /$pir-116 E. coli cells. Induction systems were cloned onto the pRSF backbone. For CRISPR/Cas9 experiments, a single RSF1010 plasmid carried both Streptococcus pyogenes Cas9 and guide RNA. dCas9 was cloned under the control of E. coli arabinose induction genes and the guide RNA under control of the constitutive J23100 promoter.

DNA yield pRSF-pLtetO-gfp was transformed via electroporation into E. coli MG1655 and V. natriegens. E. coli plates were incubated at $37^{\circ} \mathrm{C}$ and $V$. natriegens were incubated at room temperature for an equivalent time to yield approximately similar colony sizes. Three colonies from each plate was picked and grown for 5 hours at $37^{\circ} \mathrm{C}$ in $3 \mathrm{~mL}$ of selective liquid culture (LB for E. coli and LB3 for $V$. natriegens) at 225rpm. Plasmid DNA was extracted from $3 \mathrm{~mL}$ of culture (Qiagen Plasmid Miniprep Kit).

\section{CTX vibriophage infection}

$V$. cholerae $\mathrm{O} 395$ carrying the replicative form of CTX, pCTX-Km, was cultured overnight in LB without selection in a rotator drum at $150 \mathrm{rpm}$ at $30^{\circ} \mathrm{C}$. Virions were purified from cell-free supernatant $(0.22 \mu \mathrm{m}$ filtered $)$ of overnight cultures. Replicative forms were extracted from the cells by standard miniprep (Qiagen). To test infectivity of the virions, naive V. cholerae O395 and $V$. natriegens were subcultured 1:1000 in LB and LB3 respectively and mixed gently with $\sim 10^{6}$ virions. After static incubation for 30 minutes at $30^{\circ} \mathrm{C}$, the mixture was plated on selective media and incubated overnight for colony formation. Replicative forms were electroporated into host strains using described protocols.

\section{Targeted gene perturbation by Cas 9}

All Cas9 experiments were performed using a single pRSF plasmid (pRSF-paraB-Cas9-gRNA) carrying Cas9 gene under the control of arabinose promoter, with or without GFP-targeting guide RNA. All plasmids carry carbenicillin selective marker. Wild-type $V$. natriegens or strain carrying genomically integrated GFP construct were grown at $37^{\circ} \mathrm{C}$ overnight (LB3 or $\mathrm{LB} 3+100 \mu \mathrm{g} / \mathrm{ml}$ kanamycin, respectively) and transformed with 50ng of plasmid DNA using the optimized transformation protocol described above. Following 1-hour recovery in LB3 at $37^{\circ} \mathrm{C}$, cells were plated on $\mathrm{LB} 3+100 \mu \mathrm{g} / \mathrm{mL}$ carbenicillin plates and incubated overnight at $37^{\circ} \mathrm{C}$. No arabinose induction was used for Cas9 experiments, as we observed low level of baseline 
514 We transformed this engineered V. natriegens strain with a CRISPRi plasmid (pRSF-paraB515 dCas9-gRNA) carrying dCas9 under arabinose promoter and gRNA targeting GFP. To test the 516 repression of the chromosomally-encoded GFP with CRISPRi, we subcultured an overnight 517 cultures 1:1000 in fresh media supplemented with or without $1 \mathrm{mM}$ arabinose. We kinetically 518 measured $\mathrm{OD}_{600}$ and fluorescence of each culture over 12 hours in a microplate with orbital 519 shaking at $37^{\circ} \mathrm{C}$ (BioTek $\mathrm{H} 1$ or $\left.\mathrm{H} 4\right)$. Under these conditions, all cultures grew equivalently by $520 \mathrm{OD}_{600}$.

Pooled CRISPRi screen - five-member gRNA library

523 We used dCas9 (plasmid pdCas9-bacteria was a gift from Stanley Qi; Addgene plasmid \# 44249) 524 under the control of tetracycline promoter. Guide RNA was expressed under constitutive J23100 525 promoter (plasmid pCTX-R6K-gRNA). Five pCTX-R6K plasmids (spectinomycin selective marker) each carrying a gRNA were used for targeted inhibition of the following genes: $V$. natriegens targeting genes $l p t F_{V n}$ and $f \lg C_{V n}$; three control targets that do not exist in the host: E.coli gene $l p t F_{\mathrm{Ec}}$ and two GFP guides. All guides were designed to target the non-template strand. An equal mix of all five plasmids, each 20ng, was co-transformed into a dCas9 expressing $V$. natriegens strain. The transformation was recovered in $1 \mathrm{~mL}$ SOC3 media for 45 minutes at $37^{\circ} \mathrm{C}$ at $225 \mathrm{rpm}$ and plated on $245 \mathrm{~mm} \times 245 \mathrm{~mm}$ plates (Corning) with appropriate antibiotics. After 13 hours at $37^{\circ} \mathrm{C}$, colonies were scraped in LB3. Growth competition was performed by subculturing this library $1: 1000$ in LB3 at $37^{\circ} \mathrm{C}$ for 3 hours in baffled $250 \mathrm{~mL}$ flasks (Corning). At each time point, gRNA plasmid was extracted from $3 \mathrm{~mL}$ of culture (Qiagen Plasmid Miniprep Kit). Barcoded Illumina sequencing libraries were prepared by cycle-limited PCR with real-time PCR and sequenced with MiSeq v3 150. Resulting sequences were trimmed for the promoter and gRNA scaffold and the count of each guide sequence was first normalized by the number of sequences per time point, then expressed as a fraction of the sequence before growth competition.

Construction, testing, and analysis of genome-wide gRNA library

542 A custom python script was used to select gRNA sequences targeting the non-template strand of each RAST predicted protein-coding gene. Starting at the 5' end of the gene, 20bp sequences with a terminal Cas9 NGG motif on the reverse complement strand were selected. Up to 3 targets were selected for each RAST predicted gene features; each guide sequence was prefixed with a promoter and suffixed with part of the gRNA scaffold. This sequence was synthesized by the OLS process (Agilent Technologies) as an oligo library. The OLS pool was amplified by cyclelimited real-time PCR, and assembled into the pCTX-R6K-gRNA backbone (NEBuilder HiFi) at 5 -fold molar excess with $18 \mathrm{bp}$ overlap arms. $6 \mu \mathrm{L}$ of the assembled product was mixed with $300 \mu \mathrm{L}$ TransforMax EC100D pir + E. coli (Epicentre) and $51 \mu \mathrm{L}$ aliquots of this mix was electroporated in $0.1 \mathrm{~mm}$ cuvettes with a Bio-Rad Gene Pulser electroporator at $1.8 \mathrm{kV}, 200 \Omega$, $25 \mu \mathrm{F}$. These E. coli transformants were recovered in $6 \mathrm{x} 1 \mathrm{~mL}$ SOC media for 60 minutes at $37^{\circ} \mathrm{C}$ at $225 \mathrm{rpm}$, and plated on $245 \mathrm{~mm}$ x $245 \mathrm{~mm}$ spectinomycin selective plates (Corning). After 13 
554 hours at $37^{\circ} \mathrm{C}, \sim 1.4 \times 10^{6}$ colonies were scraped and plasmid DNA extracted (Qiagen HiSpeed 555 Plasmid Maxi).

The plasmid gRNA library was transformed into wild type $V$. natriegens, or a wild type strain expressing dCas9 (plasmid pdCas9-bacteria). No inducer was added for dCas9 expression, as previous results indicate leaky expression of dCas9 is sufficient for inhibition (Supplementary Figure 9) Briefly, $\sim 600 \mathrm{ng}$ of the plasmid library was mixed with $300 \mu \mathrm{L}$ of electrocompetent cells and $53.5 \mu \mathrm{L}$ of this mix was electroporated in $0.1 \mathrm{~mm}$ cuvettes with a Bio-Rad Gene Pulser electroporator at $0.4 \mathrm{kV}, 1 \mathrm{k} \Omega, 25 \mu \mathrm{F}$. Each transformation was recovered in $1 \mathrm{~mL} \mathrm{SOC} 3$ media for 45 minutes at $37^{\circ} \mathrm{C}$ at $225 \mathrm{rpm}$ and plated on $245 \mathrm{~mm} \times 245 \mathrm{~mm}$ plates (Corning) with appropriate antibiotics. After 13 hours at $37^{\circ} \mathrm{C}$, colonies were scraped in LB3 and stored at $-80^{\circ} \mathrm{C}$ as library master stocks. Pooled screens for control and test libraries (with or without the dCas9 plasmid) were performed in duplicate, starting with an initial population of $<10^{7}$ cells in $25 \mathrm{~mL}$ of media in baffled $250 \mathrm{~mL}$ flasks (Corning). For media experiments, plasmids were extracted after 8 hours of growth at $37^{\circ} \mathrm{C}$ in LB3, M9+0.4\% (w/v) Glucose, or M9+0.4\% (w/v) Sucrose. For serial passaging experiments, plasmids were extracted after 8 hours of growth at $37^{\circ} \mathrm{C}$ in LB3 (Passage 1) and the culture is then diluted 1:1000 in $25 \mathrm{~mL}$ of fresh LB3, yielding $\sim 10^{6}$ cells, and grown at $37^{\circ} \mathrm{C}$ for 4 hours (Passage 2). Subsequent passaging were performed in 4 hour increments. Plasmids were extracted from $>3 \mathrm{~mL}$ of culture (Qiagen Plasmid Miniprep Kit). Barcoded Illumina sequencing libraries were prepared by cycle-limited real-time PCR and sequenced with NextSeq v2 High Output 500/550. Resulting sequences were trimmed for the promoter and 5'end of the gRNA scaffold. Sequencing was used to verify coverage of the gRNA library. Sequencing was used to verify coverage of the gRNA library. For media experiments, the initial no dCas9 and dCas9 library contains 15,539 (99.65\%) and 13,579 (99.9\%) of 13,587 total guides, respectively. For serial passaging experiments, the initial no dCas 9 and dCas9 library contains 13,567 (99.94\%) and 13,584 (99.98\%), 13,587 total guides, respectively. After sequencing all samples, the count of each guide sequence was normalized by the number of reads per sequencing library to yield reads per million (RPM) per guide. The median gRNA copy number for the initial no dCas9 and dCas9 media library is 70.3 and 62.3 reads per million, respectively. The median gRNA copy number for the initial no dCas9 and dCas9 passaging library is 70.1 and 62.1 reads per million, respectively. These counts were input into MAGeCK and analyzed using the 'mle' module with default settings ${ }^{17}$ to obtain a fitness value (Beta score and FDR-adjusted p-value) for each gene. Essential genes from $E$. coli and $V$. cholerae were mapped to $V$. natriegens via bactNOG or COG using eggnog-mapper based on eggNOG 4.5 orthology data ${ }^{19,21,34}$. Enrichments are calculated as a one-sided hypergeometric test assuming independence between functional groups with multiple-hypothesis correction with FDR (Benjamini-Hochberg).

\section{Construction of in-frame genomic knockouts by natural competency}


593 The endogenous tfoX gene (PEG.1425) was subcloned on to the RSF1010 backbone plasmid 594 with an E. coli IPTG-inducible promoter. For each gene target, a deletion cassette, consisting of 595 an antibiotic resistance marker with 500bp homology arms upstream and downstream, was 596 created by overlap extension PCR. Generation of deletion mutants are performed as previously 597 described ${ }^{35}$. Briefly, the strain carrying tfoX is grown overnight in $1 \mathrm{mM}$ final IPTG in a rotating 598 drum at $30^{\circ} \mathrm{C}$, then subcultured $1: 100$ in $350 \mu \mathrm{L}$ of $2 \mathrm{XIO}(28 \mathrm{~g} / \mathrm{L}$ Ocean Salts) with $1 \mathrm{mM}$ IPTG 599 with $>100 \mathrm{ng}$ of the deletion cassette. The culture is incubated statically at $30^{\circ} \mathrm{C}$ for 5 hours, then 600 recovered with $1 \mathrm{~mL}$ of $\mathrm{LBv}^{2}$ at $30^{\circ} \mathrm{C}$ for 2 hours with shaking (225-250rpm). Following 601 recovery, cultures are plated on antibiotic-selective plates. Resulting transformants are screened 602 by PCR to confirm gene deletion.

603

604

605

606

616

\section{Data Availability}

Genome sequences are available in NCBI (GenBank: CP009977, CP009978; RefSeq: NZ_CP009977, NZ_CP009978). Sequencing data for gRNA counts are available in NCBI SRA under BioProject PRJNA511728 (SRR8369136, SRR8369137, SRR8369138, SRR8369139) and transcriptome data is available via GSE126544 (GSM3603279, GSM3603280, GSM3603281, GSM3603282, GSM3603283, GSM3603284). All other data are available in the Supplementary Information, or by request.

\section{Code Availability}

Custom code is available at https://github.com/citizenlee/vnat glib or will be made available upon request. 
Figure 1. Profiling of $V$. natriegens growth and genome. (a) Visualization of $V$. natriegens (Vn) and E. coli $(\mathrm{Ec})$ in a microfluidic chemostat (Cultures grown at $37^{\circ} \mathrm{C}$ in $\mathrm{LB} 3$ and $\mathrm{LB}$, respectively). Designated cell trapping heights were used to keep each species growing in a monolayer. Scale bar $10 \mu \mathrm{m}$ ( $\mathrm{N}=3$ independent experiments). (b) Single-cell growth measurement at $37^{\circ} \mathrm{C}$ (LB3 and LB, respectively). Data shown are mean $\pm \mathrm{SD}(\mathrm{N}=18$ E. coli cells and $\mathrm{N}=21 \mathrm{~V}$. natriegens cells). (c) V. natriegens chromosomes. From outside inward: two outer circles represent protein-coding genes on the plus and minus strand, respectively. Color coding by RAST annotation, as in panel $\mathrm{d}$. The third circle represents $\mathrm{G}+\mathrm{C}$ content relative to mean $\mathrm{G}+\mathrm{C}$ content of the respective chromosome, using a sliding window of 3,000 bp. tRNA and rRNA genes are shown in the fourth and fifth circles, respectively. (d) Fraction of genes in each RAST category on chr1 (dark colors) and chr2 (light yellow). (e) Filtered sequence coverage (black) and GC-skew (green) for each chromosome, as measured for exponentially growing $V$. natriegens in $\mathrm{LB} 3$ at $37^{\circ} \mathrm{C}$. Origin (red) and terminus (blue) are denoted.

Figure 2. CRISPRi screen in rich and minimal media. (a) dCas9 inhibition of chromosomallyintegrated GFP, using gRNA targeting the template $(\mathrm{T})$ or non-template (NT) strand. Inhibition was observed with (black) or without induction (gray) of dCas9. Data are shown as mean $\pm \mathrm{SD}$ ( $\mathrm{N}=4$ biological replicates). (b) Small-scale pooled CRISPRi screen. gRNAs include putative essential gene $l p t F_{V n}$ (solid black line), putative neutral genes $f l g C_{V n}$ (dashed black line), GFP (dashed gray line), and $E$. coli gene $l p t F_{E c}$ (solid gray line, target gene absent from genome). Library was grown in LB3 at $37^{\circ} \mathrm{C}$ in the presence of dCas9. Fold change in gRNA abundance is shown. (c) genome-wide gRNA library. Number (gray bars) and percentage (dotted line) of gRNAs across open reading frames are shown. (d) Schematic overview of pooled genome-wide CRISPRi screen.

Figure 3. CRISPRi inhibition in rich and minimal media. (a) Distribution of gene scores in rich and minimal media screen are shown: blue dots represent genes with significantly negative score in both rich and minimal media; red dots represent genes with significantly low score only in minimal media; gray dots represent all other genes in the population. Histogram (gray) shows distribution of all targeted genes. Inset: The number of genes with significantly negative core in each media category. (b) Single gene knock-out of sucrose-6-phosphate hydrolase located on

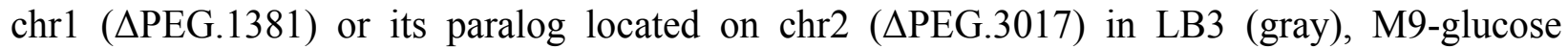
(black) or M9-sucrose (red). Data are shown as mean $\pm \mathrm{SD}(\mathrm{N}=4$ biological replicates) (c) Top ten RAST categories enriched in genes that are uniquely depleted in minimal media ( $\mathrm{N}=143$ genes). Br: Branched-Chain Amino Acid Biosynthesis, His: Histidine Biosynthesis, Bio: Biotin biosynthesis, Trp: Tryptophan synthesis, Ch: Chorismate, Au: Auxin biosynthesis, Leu: Leucine Biosynthesis, Pe: Peptide ABC transport system, Lys: Lysine Biosynthesis DAP Pathway, CoA: Co-A Biosynthesis. Statistics are derived from one-tailed hypergeometric test for enrichment 
assuming independence between subsystems. False discovery rate (FDR) controlled with Benjamini-Hochberg. (d) Leucine (L-leu) biosynthetic pathway starting from 2-pyruvate. Beta score (top) and change in expression between LB3 and M9 (fold change, bottom) are shown for each protein encoding genes (PEG number); functional genes identified by CRISPRi in minimal media are shown in red, other computationally assigned genes are shown in grey. Leucine biosynthetic genes numbered as follow: 1: acetoacetate synthase; 2: ketol-acid reductoisomerase; 3: dihydroxy-acid dehydratase; 4: isopropylmalate synthase; 5: 3-isopropylmalate dehydratase; 6: 3-isopropylmalate dehydrogenase; 7: aminotransferase.

Figure 4. Analysis of $V$. natriegens core genes. (a) Distribution of beta scores for all significantly depleted genes in rich media experiments (FDR $\leq 0.05$ ). Core genes, shown in red, display consistently negative beta score in both experiments. Negative scoring genes which were not included in core set are shown in blue (media experiment) and green (passage experiment). (b) Core genes were divided into putative essentials (yellow) and growth-supporting genes (blue). All other genes shown in gray. (c) Distribution of all targeted genes across RAST categories. Single genes, sorted by beta score, are displayed as gray lines in the respective category. Core genes shown in red. Histogram displays over-represented (filled bars) or underrepresented categories (empty bars) within all core gene set (red), putative essential genes (yellow), and growth-supporting genes (blue). Statistics are derived from one-tailed hypergeometric test for enrichment and depletion assuming independence between categories. False discovery rate (FDR) controlled with Benjamini-Hochberg. N=587 core genes. Rast category names as shown in Figure 1b. (d) Ribosomal proteins essentiality as identified in $V$. natriegens (Vn) screen. Black square denotes essential gene. Empty square denone non-essential gene. Essentiality shown for E.coli $(\mathrm{Ec})^{21}$ and V. cholerae $(\mathrm{Vc})^{19}$. LSU: Large Subunit; SSU: Small Subunit. (e) Beta score as calculated from CRISPRi screen for all gene (grey), putative essential genes (PE) and growth supporting genes (GS) are shown as a letter-value plot with median. (f) RNA-seq transcript counts as TPM (transcripts per million) for all genes (grey) and for the set of core genes, as measured at $37^{\circ} \mathrm{C}$ in LB3 medium. Putative essentials (PE) shown in yellow, growth-supporting genes (GS) shown in blue. Representative counts for a single biological replicate (out of three independent biological replicates) are shown as a letter-value plot with median. (g) Duplicated annotations of core genes. Core genes are shown as hash marks on chr1 (blue) and chr2 (green). Lines connect core genes with the respective duplicate annotation gene. Blue lines indicate core is on chr1 and duplicate on chr2. Green lines indicate core gene on chr2 and duplicate on chr1. 


\section{References}

695

696 1. Eagon, R. G. Pseudomonas natriegens, a marine bacterium with a generation time of less than 10 minutes. $J$.

$697 \quad$ Bacteriol. 83, 736-737 (1962).

698 2. Weinstock, M. T., Hesek, E. D., Wilson, C. M. \& Gibson, D. G. Vibrio natriegens as a fast-growing host for 699 molecular biology. Nat. Methods 13, 849-851 (2016).

700 3. Long, C. P., Gonzalez, J. E., Cipolla, R. M. \& Antoniewicz, M. R. Metabolism of the fast-growing bacterium 701 Vibrio natriegens elucidated by (13)C metabolic flux analysis. Metab. Eng. 44, 191-197 (2017).

702 4. Hoffart, E. et al. High substrate uptake rates empower Vibrio natriegens as production host for industrial 703 biotechnology. Appl. Environ. Microbiol. (2017). doi:10.1128/AEM.01614-17

704 5. Aiyar, S. E., Gaal, T. \& Gourse, R. L. rRNA promoter activity in the fast-growing bacterium Vibrio natriegens. $705 \quad$ J. Bacteriol. 184, 1349-1358 (2002).

706 6. Maida, I. et al. Draft Genome Sequence of the Fast-Growing Bacterium Vibrio natriegens Strain DSMZ 759. Genome Announc. 1, e00648-13-e00648-13 (2013).

708 7. Overbeek, R. et al. The SEED and the Rapid Annotation of microbial genomes using Subsystems Technology (RAST). Nucleic Acids Res. 42, D206-D214 (2013).

710 8. Thompson, J. R. \& Polz, M. F. Dynamics of Vibrio populations and their role in environmental nutrient 711 cycling. in The biology of vibrios 190-203 (American Society of Microbiology, 2006).

712 9. Heidelberg, J. F. et al. DNA sequence of both chromosomes of the cholera pathogen Vibrio cholerae. Nature $713 \quad 406,477-483(2000)$.

714 10. Chan, P. P. \& Lowe, T. M. GtRNAdb: a database of transfer RNA genes detected in genomic sequence. $715 \quad$ Nucleic Acids Res. 37, D93-7 (2009).

716 11. Brown, C. T., Olm, M. R., Thomas, B. C. \& Banfield, J. F. Measurement of bacterial replication rates in 717 microbial communities. Nat. Biotechnol. 34, 1256-1263 (2016).

718 12. van Opijnen, T. \& Camilli, A. Genome-wide fitness and genetic interactions determined by Tn-seq, a high719 throughput massively parallel sequencing method for microorganisms. Curr. Protoc. Microbiol. Chapter 1, $720 \quad$ Unit1E.3 (2010).

721 13. Rock, J. M. et al. Programmable transcriptional repression in mycobacteria using an orthogonal CRISPR 
interference platform. Nat Microbiol 2, 16274 (2017).

723 14. Peters, J. M. et al. A Comprehensive, CRISPR-based Functional Analysis of Essential Genes in Bacteria. Cell

$724 \quad \mathbf{1 6 5}, 1493-1506(2016)$.

725 15. Qi, L. S. et al. Repurposing CRISPR as an RNA-guided platform for sequence-specific control of gene expression. Cell 152, 1173-1183 (2013).

727 16. Liu, X. et al. High-throughput CRISPRi phenotyping identifies new essential genes in Streptococcus 728 pneumoniae. Mol. Syst. Biol. 13, 931 (2017).

729 17. Li, W. et al. MAGeCK enables robust identification of essential genes from genome-scale CRISPR/Cas9 $730 \quad$ knockout screens. Genome Biol. 15, 554 (2014).

731 18. Tatusova, T. et al. NCBI prokaryotic genome annotation pipeline. Nucleic Acids Res. 44, 6614-6624 (2016).

732 19. Chao, M. C. et al. High-resolution definition of the Vibrio cholerae essential gene set with hidden Markov 733 model-based analyses of transposon-insertion sequencing data. Nucleic Acids Res. 41, 9033-9048 (2013).

734 20. Okada, K., Iida, T., Kita-Tsukamoto, K. \& Honda, T. Vibrios commonly possess two chromosomes. J.

$735 \quad$ Bacteriol. 187, 752-757 (2005).

736 21. Yamazaki, Y., Niki, H. \& Kato, J.-I. Profiling of Escherichia coli Chromosome database. Methods Mol. Biol. $737 \quad 416,385-389(2008)$.

738 22. Julio, S. M. et al. DNA Adenine Methylase Is Essential for Viability and Plays a Role in the Pathogenesis of Yersinia pseudotuberculosis andVibrio cholerae. Infect. Immun. 69, 7610-7615 (2001).

740 23. Egan, E. S., Fogel, M. A. \& Waldor, M. K. Divided genomes: negotiating the cell cycle in prokaryotes with 741 multiple chromosomes. Mol. Microbiol. 56, 1129-1138 (2005).

742 24. Wang, T. et al. Identification and characterization of essential genes in the human genome. Science 350, 1096$743 \quad 1101(2015)$.

744 25. Schleicher, L. et al. Vibrio natriegens as Host for Expression of Multisubunit Membrane Protein Complexes. $745 \quad$ Front. Microbiol. 9, 2537 (2018).

746 26. Lenz, D. H. et al. The small RNA chaperone Hfq and multiple small RNAs control quorum sensing in Vibrio 747 harveyi and Vibrio cholerae. Cell 118, 69-82 (2004).

748 27. Val, M.-E. et al. A checkpoint control orchestrates the replication of the two chromosomes of Vibrio cholerae. $749 \quad$ Sci Adv 2, e1501914 (2016). 
28. Mee, M. T., Collins, J. J., Church, G. M. \& Wang, H. H. Syntrophic exchange in synthetic microbial communities. Proc. Natl. Acad. Sci. U. S. A. 111, E2149-56 (2014).

752 29. Cui, L. et al. A CRISPRi screen in E. coli reveals sequence-specific toxicity of dCas9. Nat. Commun. 9, 1912 $753 \quad$ (2018).

754 30. Zhang, S. \& Voigt, C. A. Engineered dCas9 with reduced toxicity in bacteria: implications for genetic circuit 755 design. Nucleic Acids Res. 46, 11115-11125 (2018).

756 31. Vega, N. M., Allison, K. R., Khalil, A. S. \& Collins, J. J. Signaling-mediated bacterial persister formation. Nat. Chem. Biol. 8, 431-433 (2012).

32. Martínez-García, E., Calles, B., Arévalo-Rodríguez, M. \& de Lorenzo, V. pBAM1: an all-synthetic genetic tool for analysis and construction of complex bacterial phenotypes. BMC Microbiol. 11, 38 (2011).

33. Cameron, D. E., Urbach, J. M. \& Mekalanos, J. J. A defined transposon mutant library and its use in identifying motility genes in Vibrio cholerae. Proc. Natl. Acad. Sci. U. S. A. 105, 8736-8741 (2008).

34. Huerta-Cepas, J. et al. Fast genome-wide functional annotation through orthology assignment by eggNOGmapper. Mol. Biol. Evol. (2017). doi:10.1093/molbev/msx148

35. Dalia, T. N. et al. Multiplex Genome Editing by Natural Transformation (MuGENT) for Synthetic Biology in Vibrio natriegens. ACS Synth. Biol. (2017). doi:10.1021/acssynbio.7b00116 

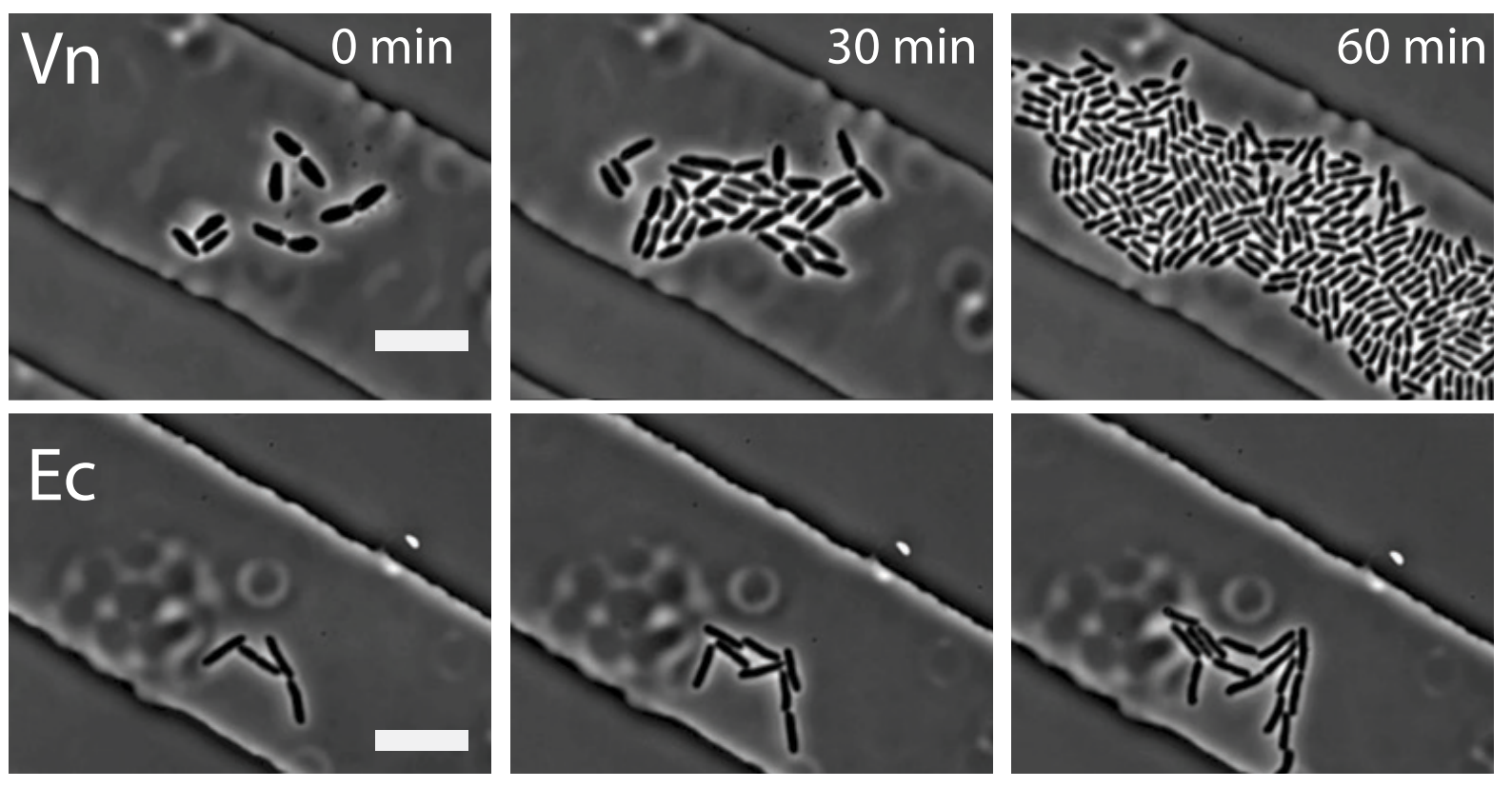

C
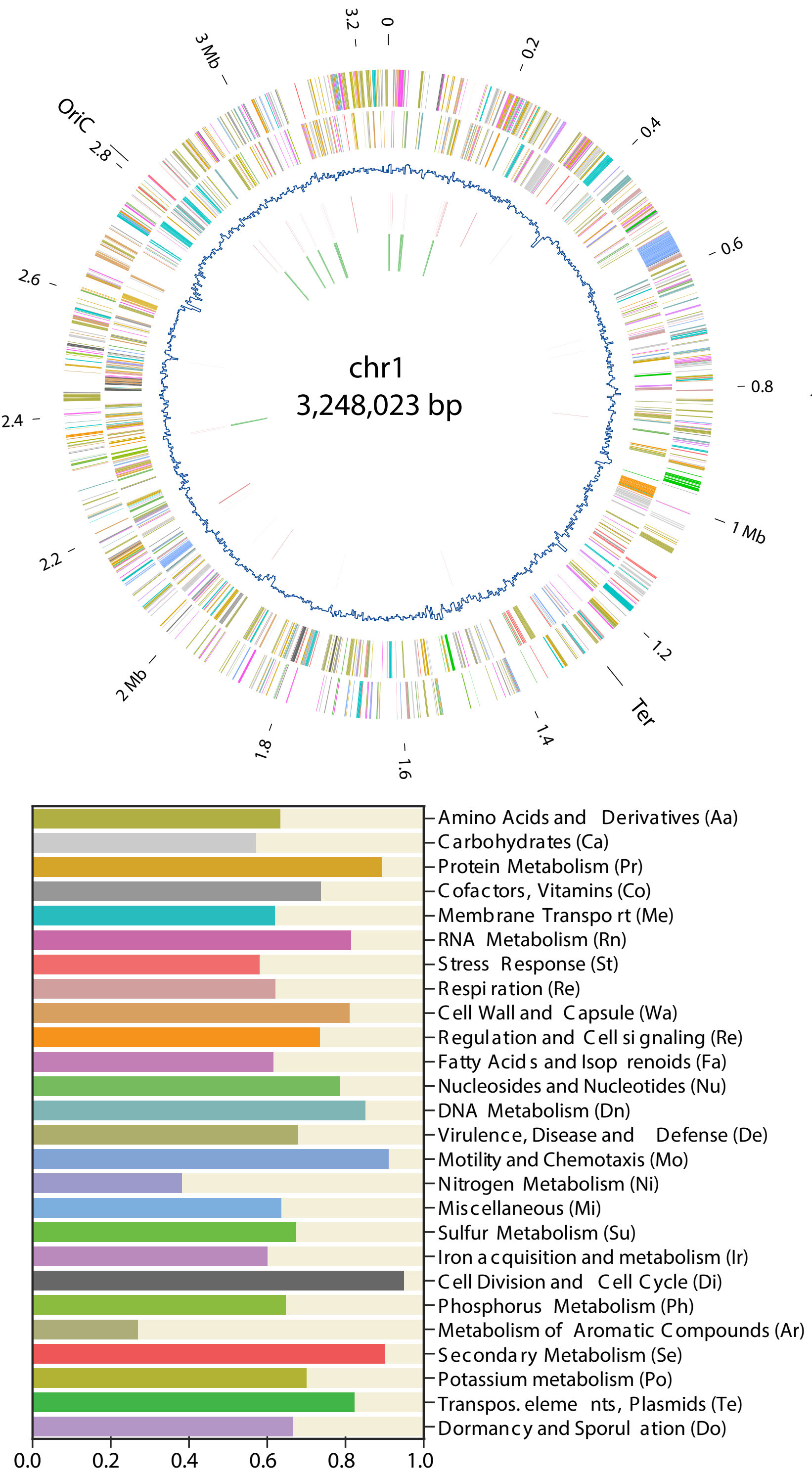

Fraction of genes in category

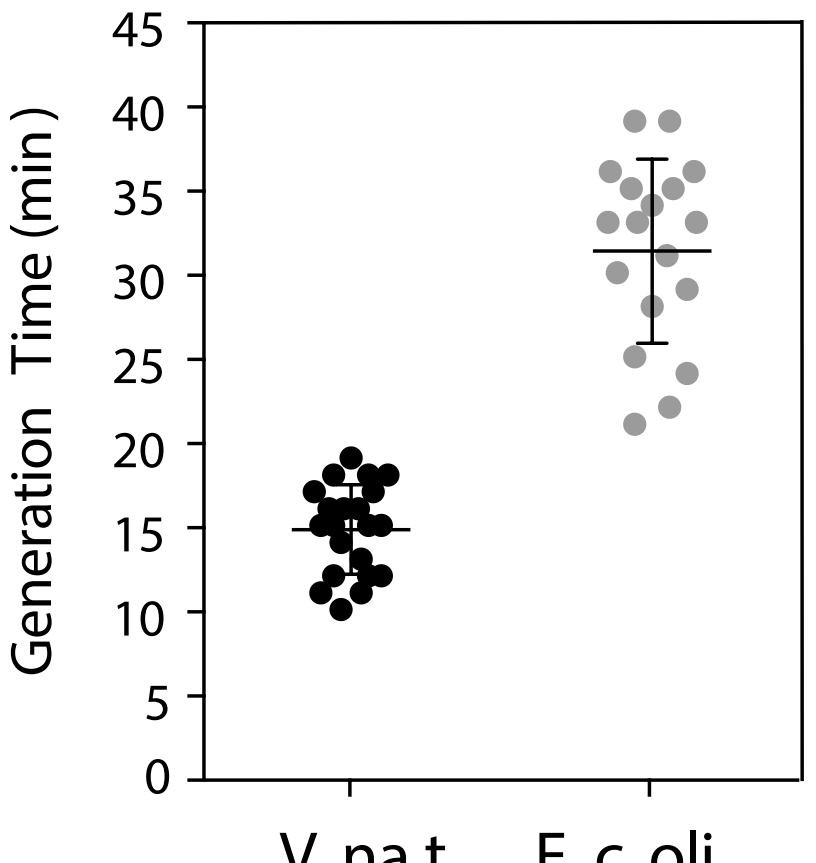

V. na t E.c oli

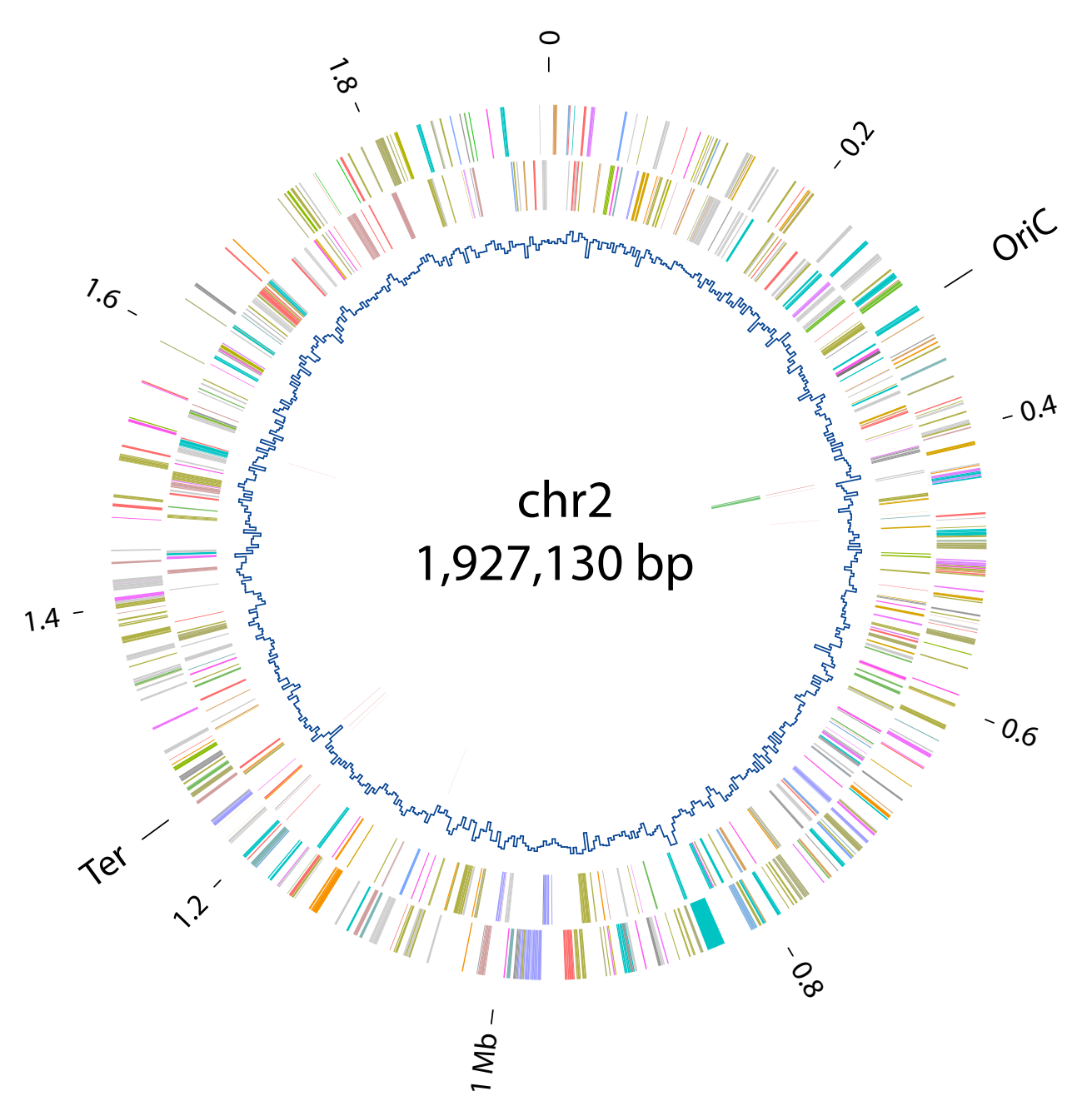

e

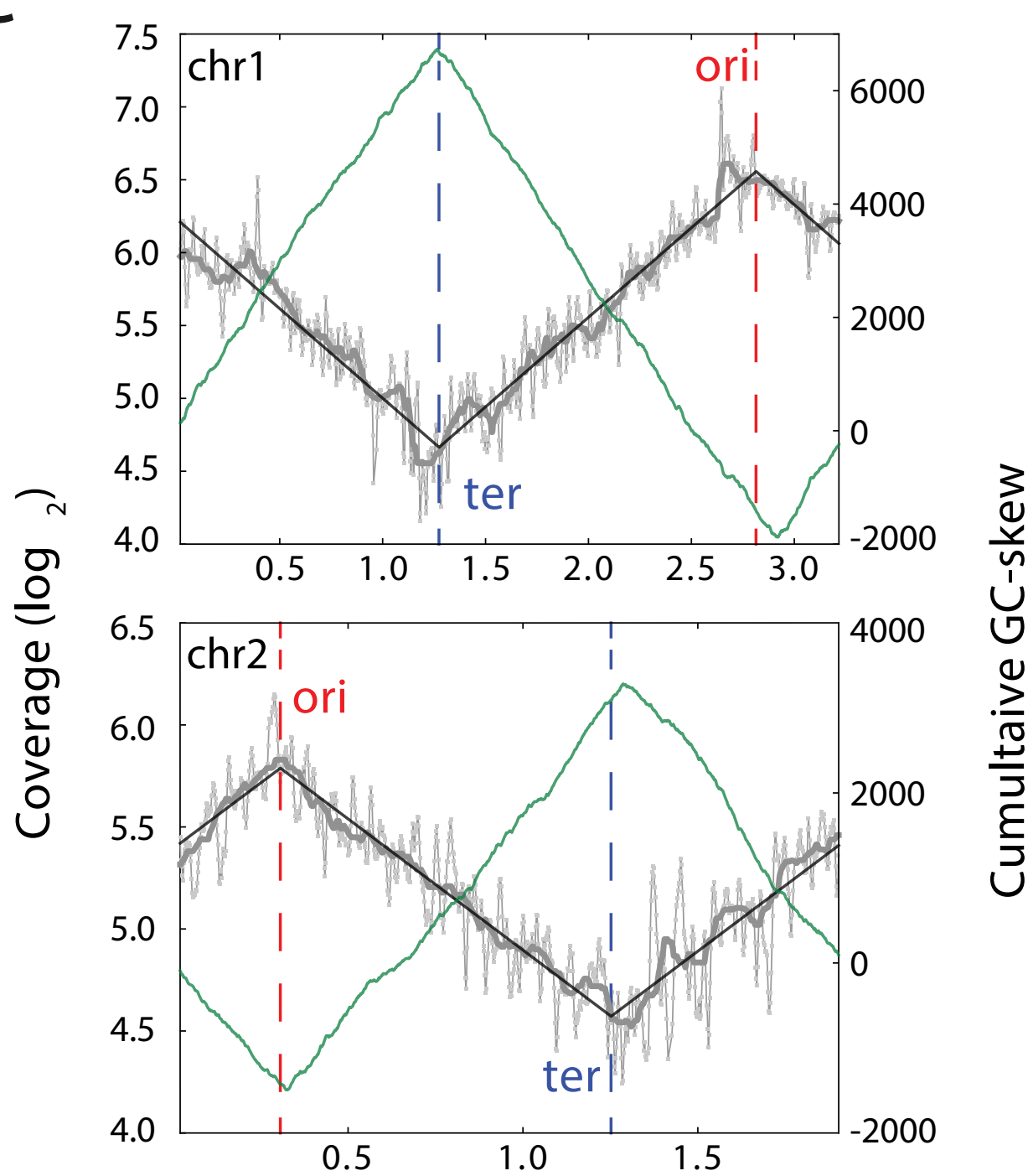

Position on Genome (Mb) 

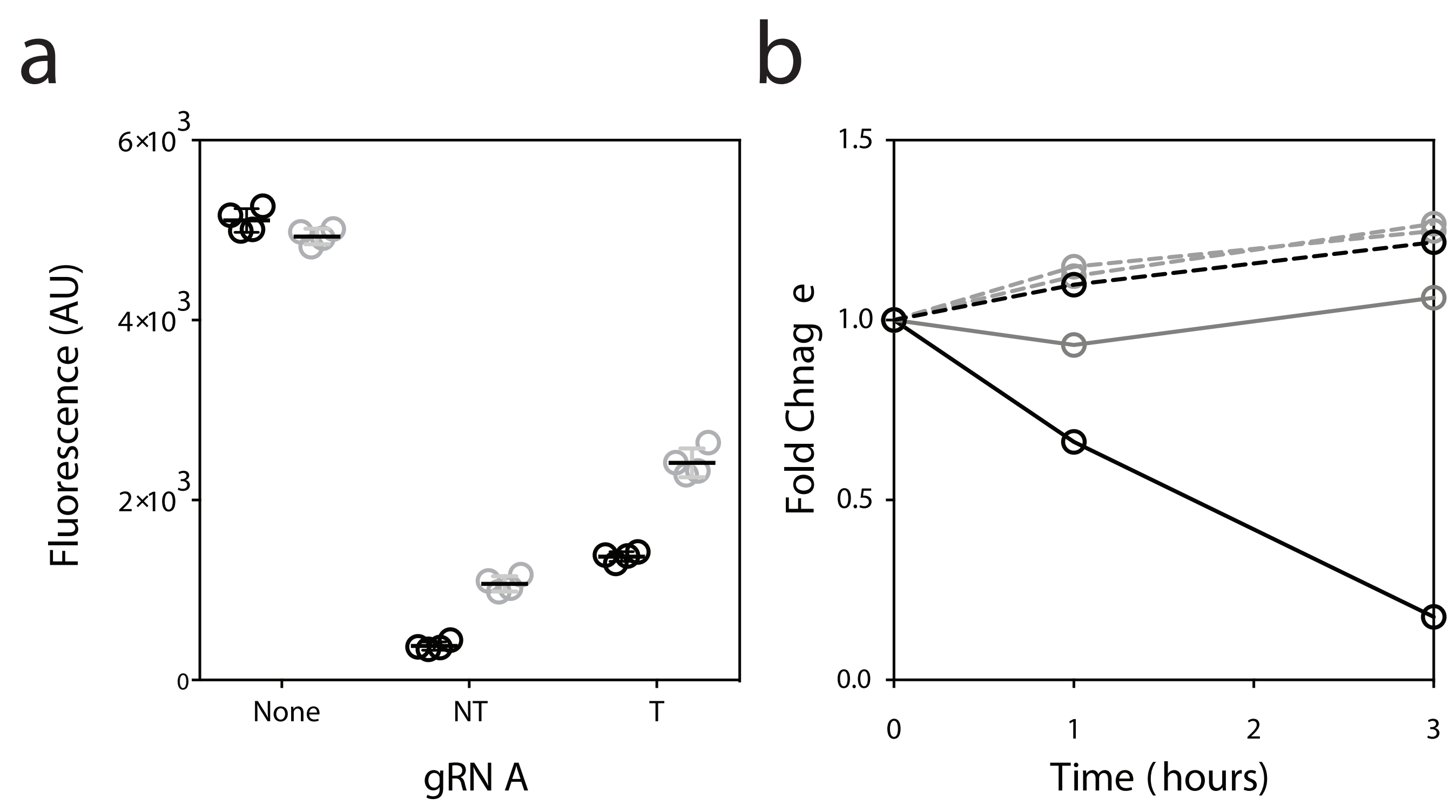

d

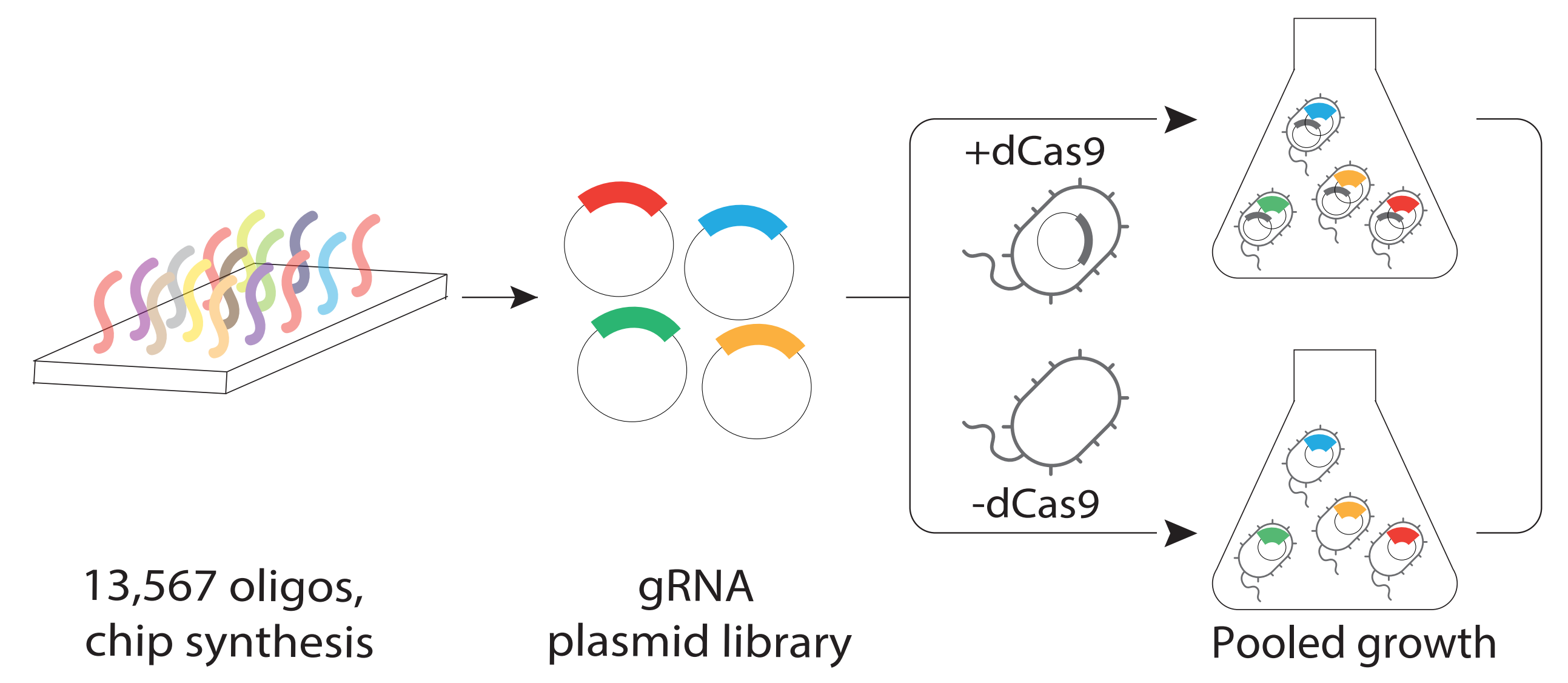

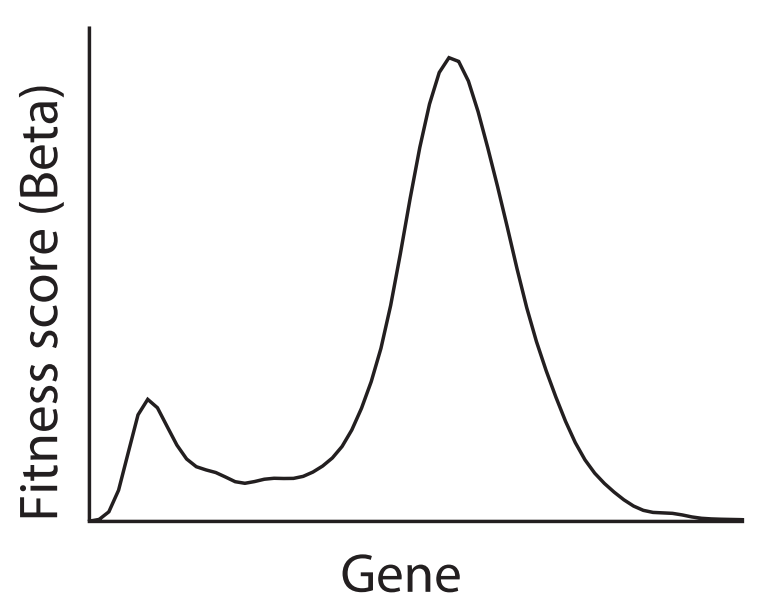

MAGeCK analysis:

Beta score per gene

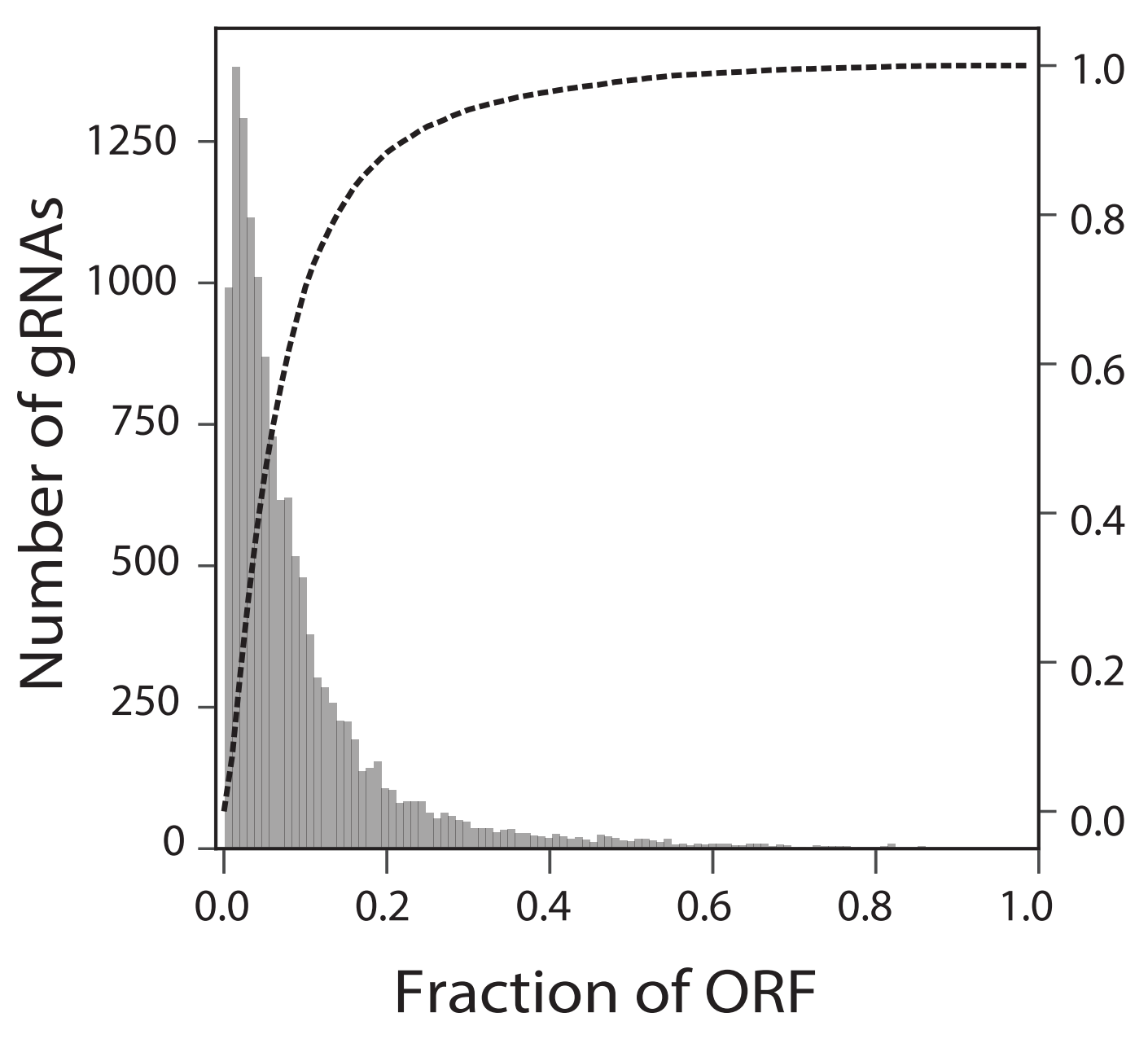

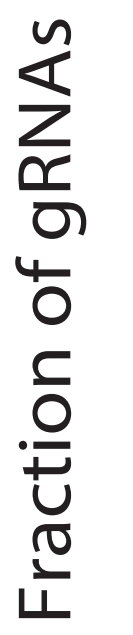

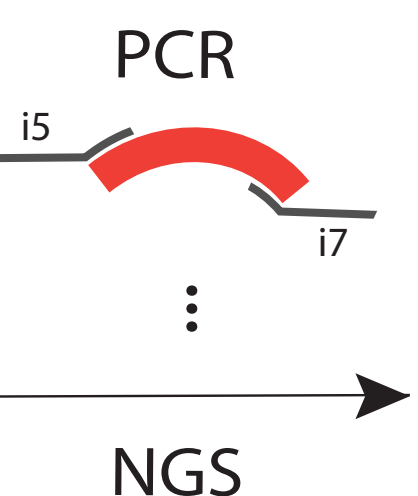


a

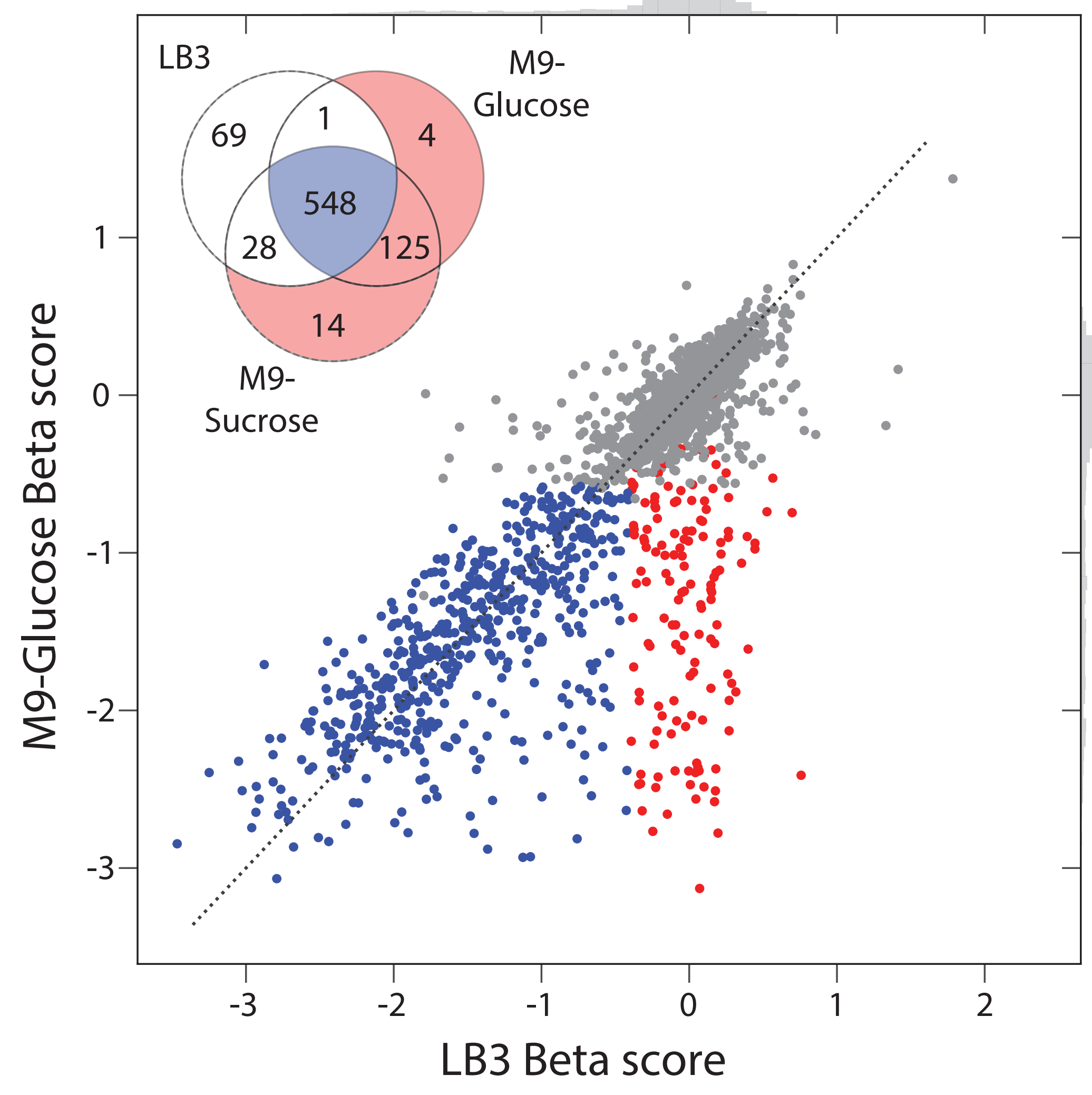

b

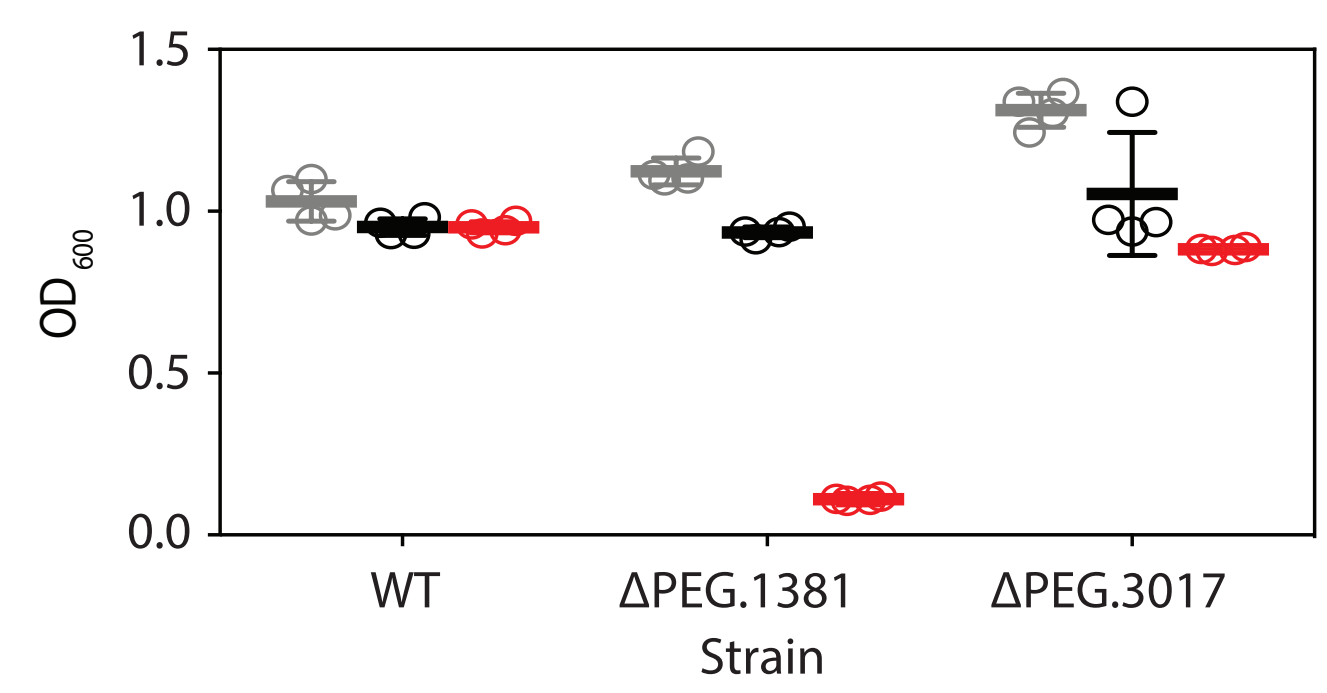

C

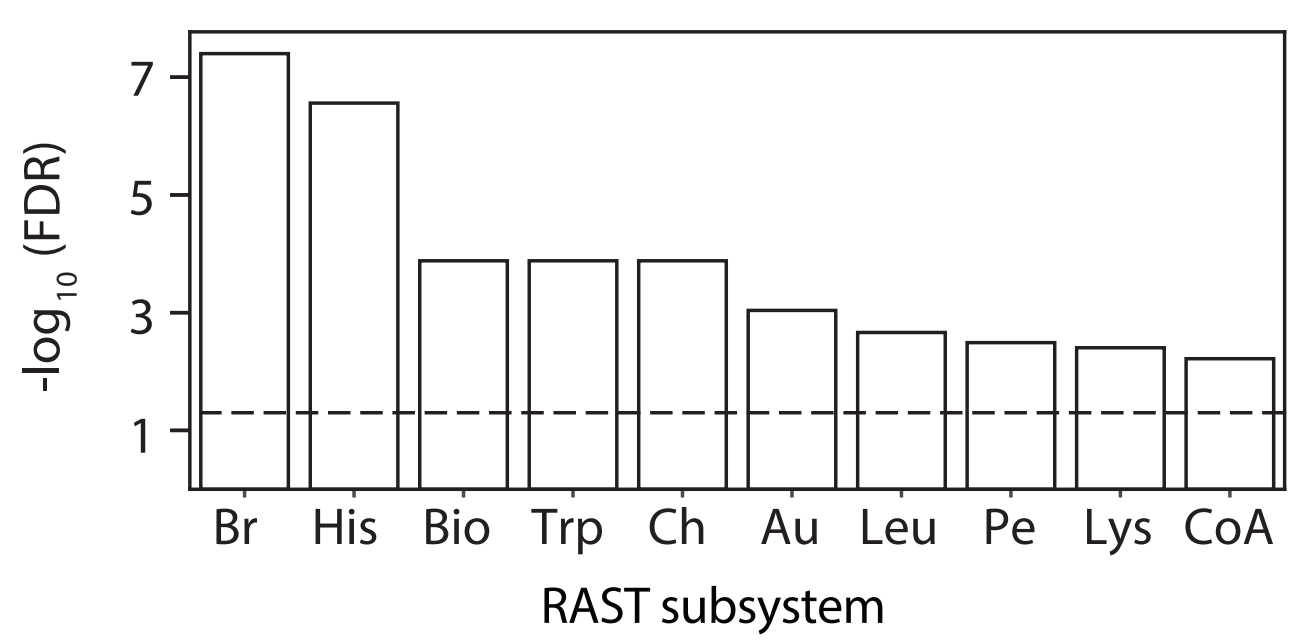

d

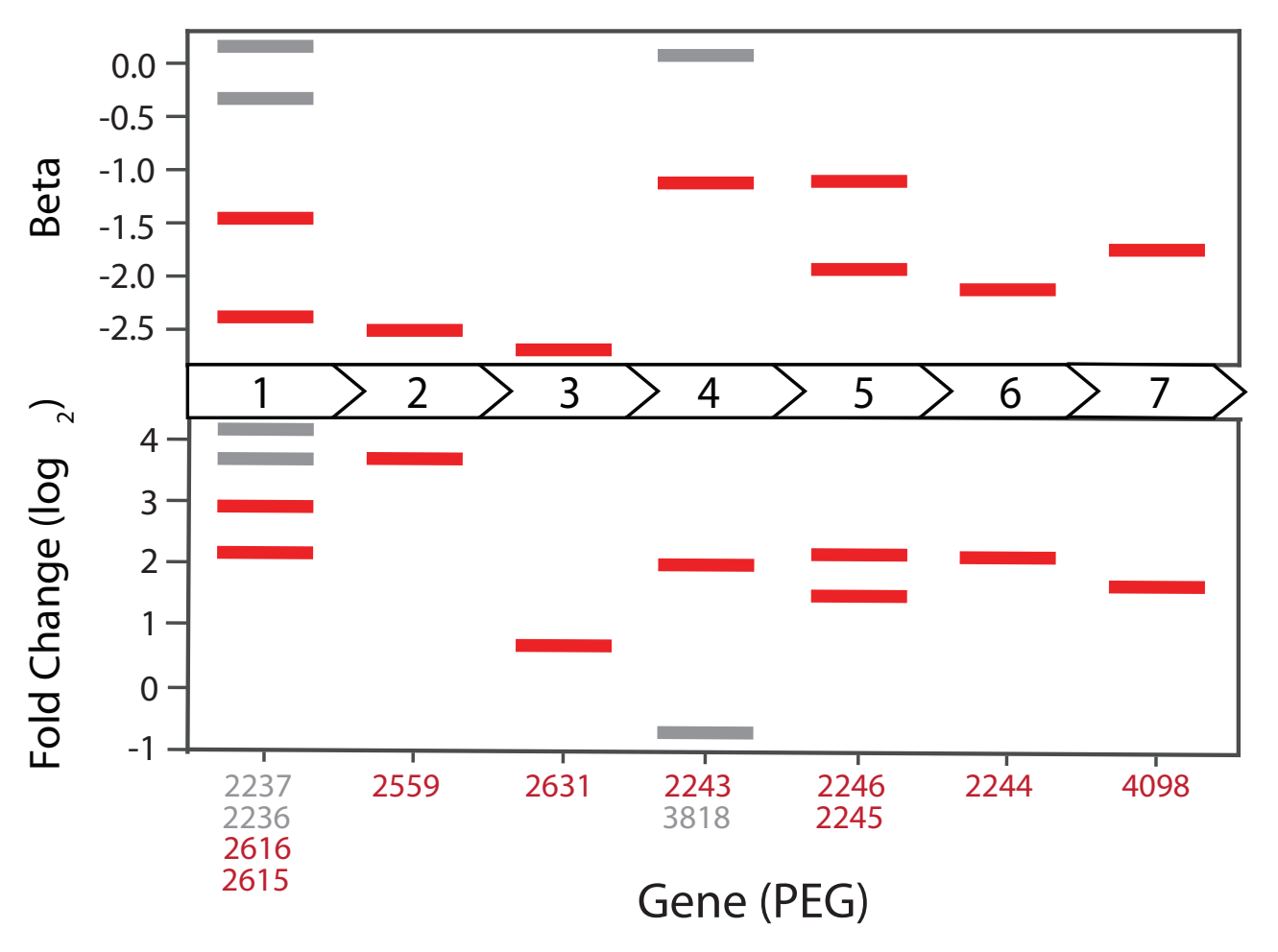


a

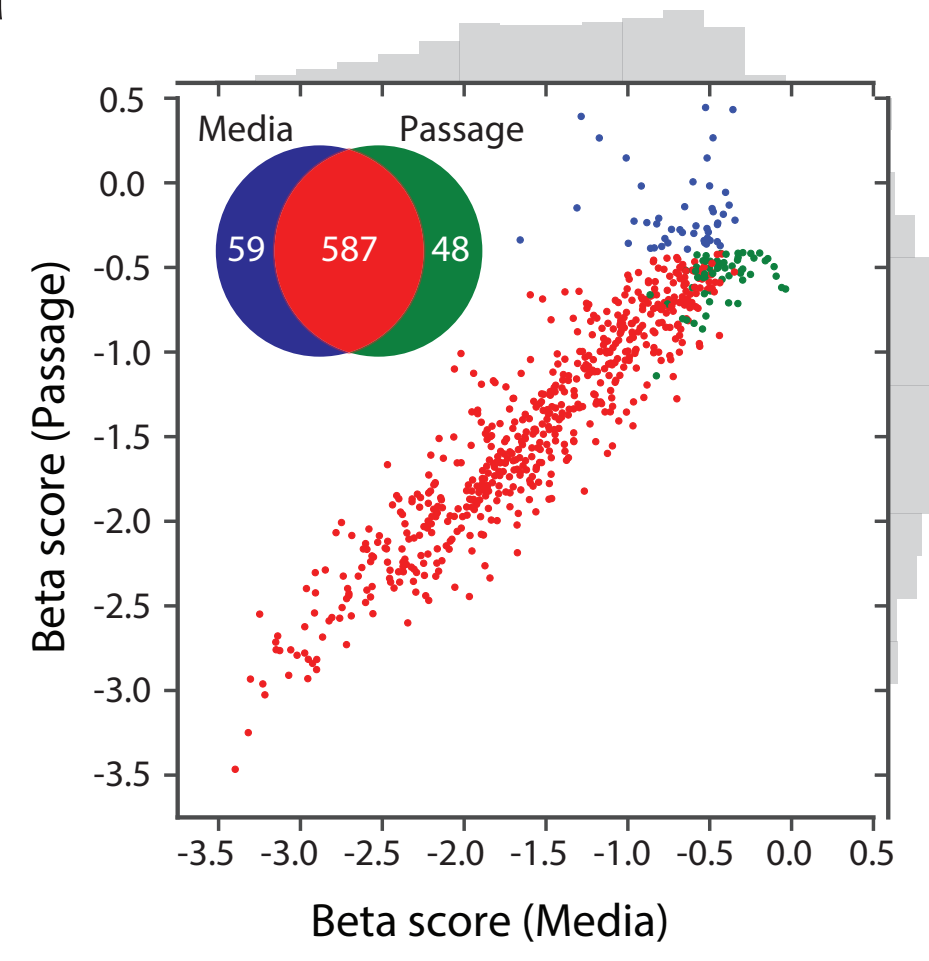

b

Putative Essential (278 genes)

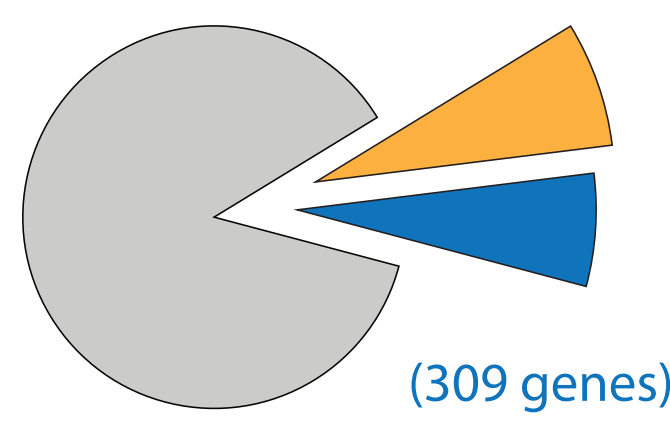

Growth supporting

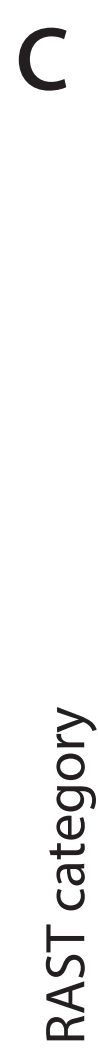

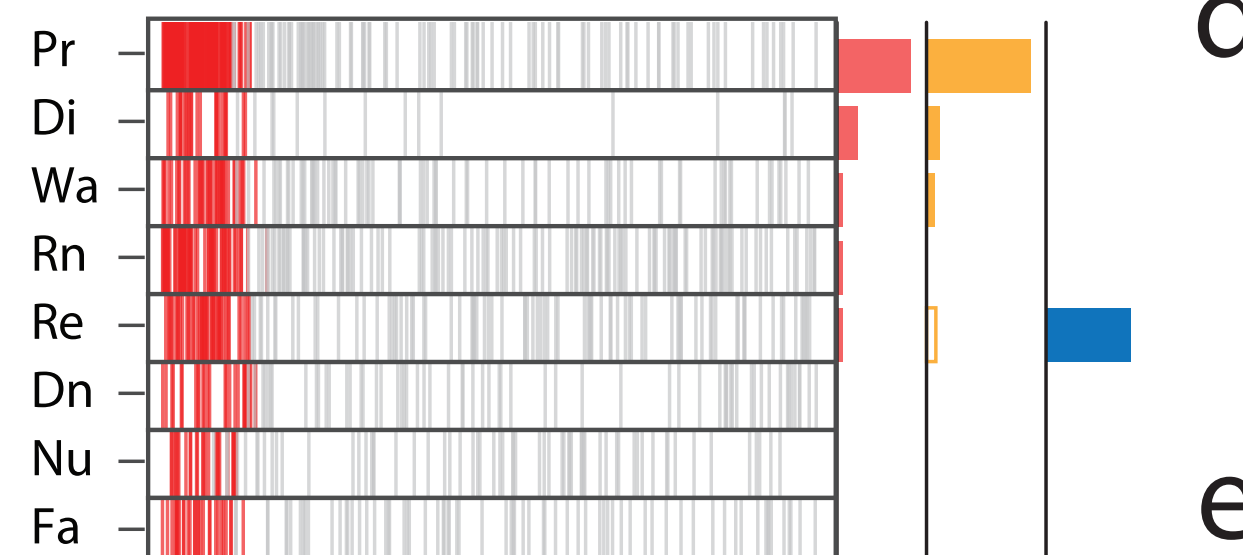

Do

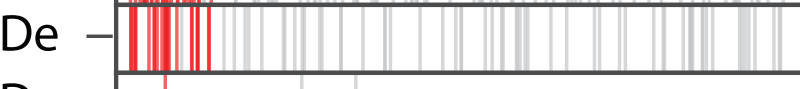

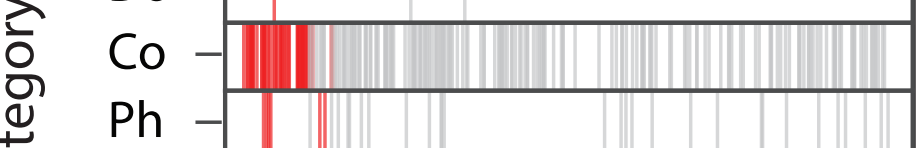

造

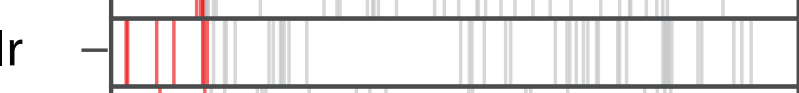

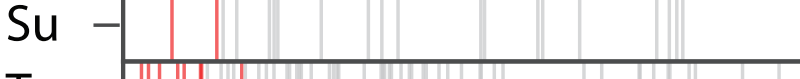

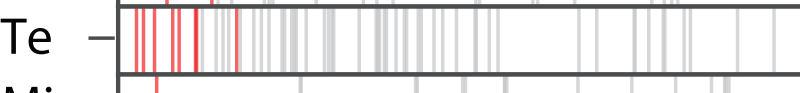

Mi -

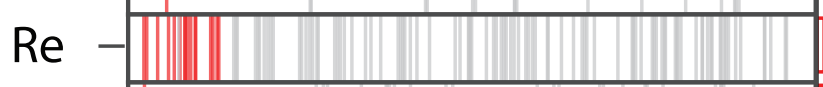

Ar -

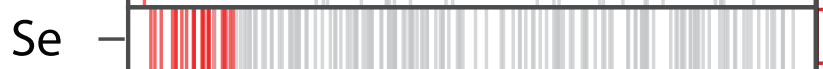

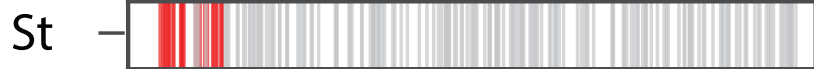

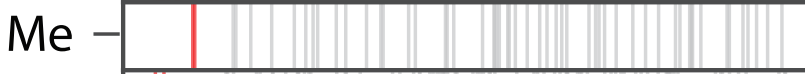

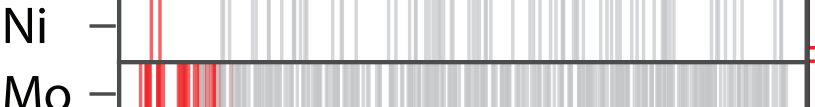

Mo

$\mathrm{Ca}$

Aa

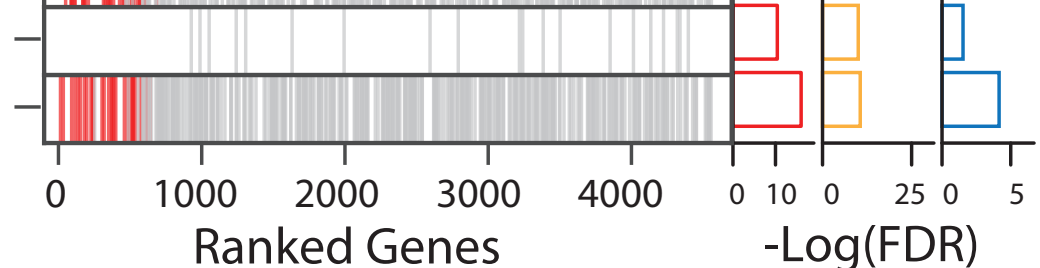

rpm rpl

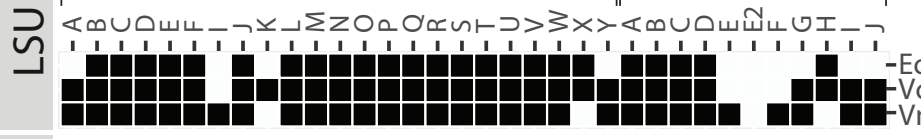

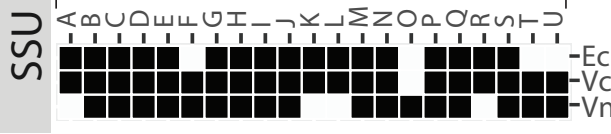

e

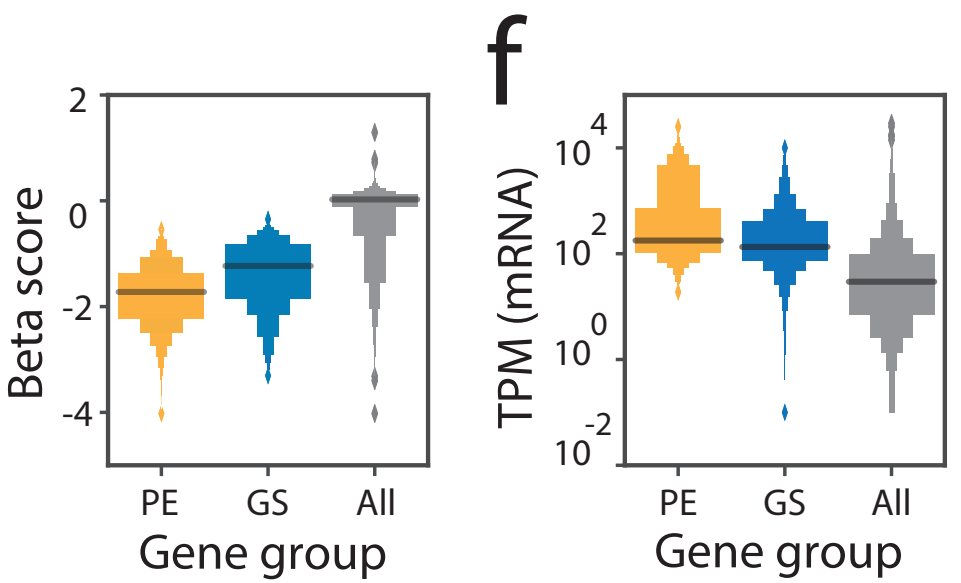

9

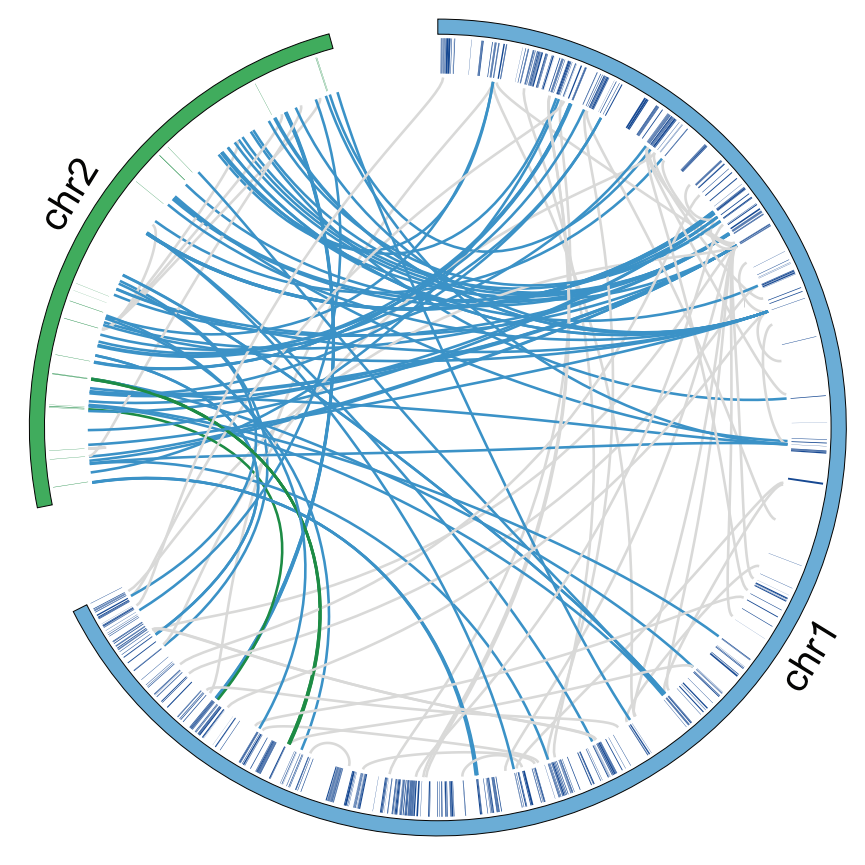

